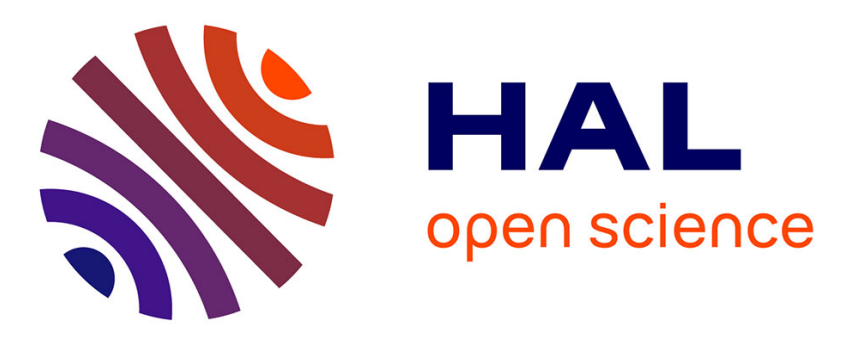

\title{
Multi-scale investigation in the frequency domain of Ar/HMDSO dusty plasma with pulsed injection of HMDSO
}

V Garofano, Remi Berard, S Boivin, C. Joblin, Kremena Makasheva, L. Stafford

\section{To cite this version:}

V Garofano, Remi Berard, S Boivin, C. Joblin, Kremena Makasheva, et al.. Multi-scale investigation in the frequency domain of Ar/HMDSO dusty plasma with pulsed injection of HMDSO. Plasma Sources Science and Technology, 2019, 28 (5), pp.055019. 10.1088/1361-6595/ab07cc . hal-02332813

\section{HAL Id: hal-02332813 https://hal.science/hal-02332813}

Submitted on 25 Oct 2019

HAL is a multi-disciplinary open access archive for the deposit and dissemination of scientific research documents, whether they are published or not. The documents may come from teaching and research institutions in France or abroad, or from public or private research centers.
L'archive ouverte pluridisciplinaire HAL, est destinée au dépôt et à la diffusion de documents scientifiques de niveau recherche, publiés ou non, émanant des établissements d'enseignement et de recherche français ou étrangers, des laboratoires publics ou privés. 


\title{
Multi-scale investigation in the frequency domain of Ar/HMDSO dusty plasma with pulsed injection of HMDSO
}

\author{
V Garofano ${ }^{1}$, R Bérard ${ }^{2,3}$, S Boivin ${ }^{1}$, C Joblin $^{3}$, K Makasheva $^{2,4}$ (1) and \\ L Stafford ${ }^{1,4}$ (i) \\ ${ }^{1}$ Département de physique, Université de Montréal, Montréal, Québec, Canada \\ ${ }^{2}$ LAPLACE (Laboratoire Plasma et Conversion d'Energie), Université de Toulouse, CNRS, UPS, INPT, \\ Toulouse, France \\ ${ }^{3}$ IRAP (Institut de Recherche en Astrophysique et Planétologie), Université de Toulouse, CNRS, UPS, \\ CNES, Toulouse, France
}

E-mail: kremena.makasheva@laplace.univ-tlse.fr and luc.stafford@umontreal.ca

Received 30 August 2018, revised 16 January 2019

Accepted for publication 18 February 2019

Published 24 May 2019

\begin{abstract}
A combination of time-resolved optical emission spectroscopy measurements and collisionalradiative modeling is used to investigate the phenomena occurring over multiple time scales in the frequency domain of a low-pressure, axially asymmetric, capacitively coupled radiofrequency $(\mathrm{RF})$ argon plasma with pulsed injection of hexamethyldisiloxane (HMDSO,

$\left.\mathrm{Si}_{2} \mathrm{O}\left(\mathrm{CH}_{3}\right)_{6}\right)$. The collisional-radiative model developed here considers the population of argon 1s and all ten $2 p$ levels (in Paschen's notation). The presence of HMDSO in the plasma is accounted for in the model by quenching of the argon $1 \mathrm{~s}$ states by species generated by plasma processing of HMDSO, including HMDSO-15 $\left(\mathrm{Si}_{2} \mathrm{O}\left(\mathrm{CH}_{3}\right)_{5}\right)$, acetylene $\left(\mathrm{C}_{2} \mathrm{H}_{2}\right)$ and methane $\left(\mathrm{CH}_{4}\right)$. Detailed analysis of the relative populations of Ar $2 \mathrm{p}$ states reveals cyclic evolutions of the electron temperature, electron density and quenching frequency that are shown to be linked to the kinetics of dust formation in Ar/HMDSO plasmas. Penning ionization of HMDSO and its fragments is found to be an important source of electrons for the plasma maintenance. It is at the origin of the cyclic formation/disappearance of the dust cloud, without attenuation of the phenomenon, as long as the pulsed injection of HMDSO is sustained. The multi-scale approach used in this study further reveals the straightforward relation of the frequency of HMDSO pulsed injection, in particular the HMDSO duty cycle, with the frequency of dust formation/

disappearance cycle.
\end{abstract}

Keywords: dusty plasmas, optical emission spectroscopy, electron temperature, electron density, HMDSO plasmas

\section{Introduction}

Plasmas containing nano-sized particles, or more generally, dusty plasmas, can be generated through numerous processes, the most widespread ones being: (i) simple injection of particles through dispensers usually situated at the electrodes [1], (ii) spray injection into the plasma of colloidal solutions containing nanoparticles [2, 3], (iii) formation of metallic nanocrystals or dielectric nanoparticles by sputtering of a target [4-7] and (iv) growth of dust in a reactive plasma from the working gas mixture [8-25]. Although a number of reactive gases (particularly $\mathrm{SiH}_{4}$, $\mathrm{C}_{2} \mathrm{H}_{2}$ and $\mathrm{CH}_{4}$ ) have been used in the study of dust growth dynamics in reactive plasmas [8-20], a lot of other reactive agents can form nanoparticles. For example, large molecules such as hexamethyldisiloxane (HMDSO, $\mathrm{Si}_{2} \mathrm{O}\left(\mathrm{CH}_{3}\right)_{6}$, mass $162.38 \mathrm{~g} \mathrm{~mol}^{-1}$ ) [21-23], tetraethyl orthosilicate (TEOS, $\mathrm{Si}\left(\mathrm{OC}_{2} \mathrm{H}_{5}\right)_{4}$, mass $\left.208.33 \mathrm{~g} \mathrm{~mol}^{-1}\right)$ [24] and aniline $\left(\mathrm{C}_{6} \mathrm{H}_{5}\left(\mathrm{NH}_{2}\right)\right.$, mass $93.13 \mathrm{~g} \mathrm{~mol}^{-1}$ ) [25] have been identified as precursors 
yielding dusty plasma. Their injection in the plasma can be continuous or pulsed, often leading to the succession of tens or hundreds of generations of dust for the pulsed injection [22]. The interest in using large molecular precursors in plasma processes is supported by their current and potential applications in microelectronics, optics and energy storage devices or as barrier films in food packaging and flame retardant layers [26-30], etc. However, special attention should be paid to their toxicity level and accordingly, to the general rules for safety use. In contrary to HMDSO, which represents a rare case of a safe, non-toxic and non-flammable agent, the use of aniline should be accompanied by a strict safety procedure due to its high toxicity level and easily flammable state. With respect to dusty plasmas, the fact that these molecules are quite large compared to classical dust precursors means that there is a plethora of possible bond breaking and recombination reactions, opening the door to many different scenarios for the plasma composition and the formation of nano-sized particles in the plasma.

One can state that dusty plasmas represent a particular class of gas discharges, where the physical phenomena occur in a very broad frequency domain. As observed in many types of dusty plasmas, the growth of nanoparticles in the gas phase is subject to temporal and spatial variations. Steady-state conditions are generally hard to reach because the balance of the various forces acting on the nanoparticles to maintain them in the gas phase significantly varies with their formation and loss dynamics. In addition, the formation of dusty plasmas largely depends on the experimental conditions and the presence of instabilities in the discharge. A particular example for the dynamic character of instabilities in dusty plasmas is the development of a dust-free region, called a void [1, 22, 31, 32]. The heart-beat frequency of the void is found to be in the range of few $\mathrm{Hz}$ [32]. New generations of dust are suspected to start growing in the void, which appears in the central part of the discharge pushing previously formed bigger dust particles towards the plasma edge. It is common in dusty plasmas to find the frequency of appearance of successive generations of dust in the $\mathrm{mHz}$ range [22, 33]. Thus, for completeness, the analysis of the dynamic behaviour of dusty plasmas requires consideration of as many processes occurring in the plasma as possible, particularly in the case of pulsed injection of the reactive gas.

A large number of plasma diagnostic methods have been adapted or specially developed to investigate different aspects of the complex phenomena occurring in dusty plasmas. Measurements of the electrical properties of dusty plasmas allowed detection of the changes in the plasma state in the presence of dust. The time evolution of the fundamental frequency and harmonics of the current was followed with the intention to probe the formation of very small-less than 5 $\mathrm{nm}$-particles in the plasma [34, 35]. Measuring the self-bias and floating potentials in radio-frequency (RF) discharges can provide additional information on the dynamical behaviour of dusty plasmas, indicating the tendency to a more electronegative plasma state in the presence of dust [20, 22, 33]. Measurements of the mean electron energy and electron density were achieved with Langmuir probes, which completes the understanding of the effects of dust particles on the electron energy probability function (EEPF) in these plasmas
$[7,36]$. The signature of the precursors, the precursor depletion in the plasma and the growth mechanisms of dust were revealed by in situ Fourier transform infrared (FTIR) spectroscopy [37-39]. For hydrocarbon dusty plasmas, two groups of large absorption bands in the FTIR spectrum, attributed to $s p^{1}$ species and to substituted $s p^{2}$ aromatic rings, were identified in the presence of dust inside the plasma [38]. Plasma sampling mass spectrometry has been used to detect the potential precursors of dust particles in plasmas of methane, acetylene, ethylene and HMDSO [19, 23, 39]. A large production of acetylenic compounds $\left(\mathrm{C}_{2} \mathrm{H}_{\mathrm{x}}\right)$, considered to be responsible for the production of species involved in dust formation channels independently from the investigated hydrocarbon plasmas, was constantly reported. Diagnostic methods based on laser light scattering (LLS) methods using multiple lasers or light extinction spectroscopy [4, 18, 40-43] have also been used to extract information on the dust size and density or to map the dust cloud.

Optical emission spectroscopy (OES) remains a very suitable, non-intrusive, diagnostic method to study the evolution of the electron population and the electron mean energy in dusty plasmas. However, the application of OES in dusty plasmas should be cautiously performed, and the theoretical model for interpretation of the recorded spectra should account for the chemistry of the reactive gas. Studies reporting the application of OES in dusty RF capacitively coupled discharges sustained in mixtures of $\mathrm{Ar} / \mathrm{H}_{2} / \mathrm{C}_{2} \mathrm{H}_{2}$ and $\mathrm{Ar} / \mathrm{H}_{2} / \mathrm{CH}_{4}$ [44], $\mathrm{CH}_{4} / \mathrm{H}_{2}$ and $\mathrm{C}_{3} \mathrm{H}_{8} / \mathrm{H}_{2}$ [45], and $\mathrm{Ar} / \mathrm{N}_{2} / \mathrm{CH}_{4}[46,47]$ are based on the socalled corona model. In this model, excitation of emitting states exclusively occurs by electron impact on ground state atoms; stepwise excitation by electron impact and cascading effects, as well as the quenching of excited states by heavy particles, are completely neglected. In some studies, the mean electron energy is extracted, as a function of the applied power or gas pressure, on the basis of modified Boltzmann plots or using the line ratio technique, considering the intensities of $\mathrm{H}_{\alpha}, \mathrm{H}_{\beta}$ and $\mathrm{H}_{\gamma}$ lines for discharges sustained in pure hydrocarbon gases or the intensities of different Ar-lines when argon is used as the carrier gas in the mixture. It is generally established that the time evolution of $T_{\mathrm{e}}$ follows the variations in the intensity of Ar-lines in a consistent way with the generation of dust in the plasma [47]. Recently, an initial study of the time evolution of the mean electron energy in $\mathrm{Ar} / \mathrm{HMDSO}$ plasmas, based on trace-rare-gases optical emission spectroscopy (TRG-OES) coupled with a collisionalradiative model was reported in the literature [48]. After elaboration of a Boltzmann diagram for the Ar high-lying levels and a collisional-radiative model for the $\mathrm{Xe}, \mathrm{Kr}$ and $\mathrm{Ar} 2 \mathrm{p}$ (Paschen notation) states, temperatures characterizing the lowand high-energy parts of the electron energy probability function were calculated. The obtained results show a cyclic evolution of these temperatures, with an increase when dust nanoparticles grow in the plasma and a decrease when they are lost.

In this work a combination of time-resolved OES measurements and collisional-radiative modeling is used to probe phenomena occurring over multiple time scales in the frequency domain in a low-pressure, axially asymmetric, capacitively coupled RF argon plasma with pulsed injection of HMDSO. The objective is to simultaneously track the main 

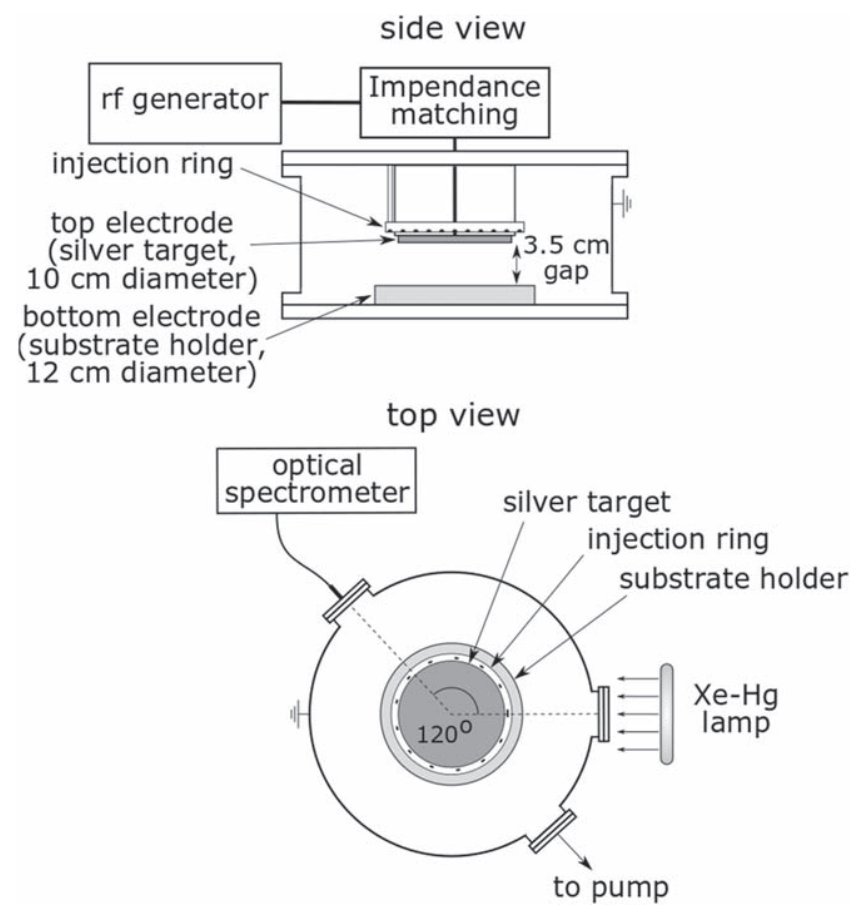

Figure 1. A sketch of the experimental setup.

plasma parameters, i.e. mean electron energy and electron density, considering the multi-scale action of different physical phenomena occurring in the dusty plasma. Special attention is paid to the quenching of argon metastable species in the plasma. The working hypothesis is the following: the HMDSO and the following plasma-generated species efficiently quench the argon metastable atoms, which in turn provoke alteration in the stepwise excitation of emitting Ar $2 p$ states probed by OES. The combination of OES and collisional-radiative modelling can thus be used to judiciously analyze variations in the mean electron energy, electron density and quenching frequency of $\mathrm{Ar} 1 \mathrm{~s}$ states during the cyclic formation/disappearance of dust.

\section{Materials and methods}

\subsection{Description of the plasma reactor}

The plasma consists of an axially asymmetric capacitively coupled RF discharge sustained at $13.56 \mathrm{MHz}$ at low pressure $(<10 \mathrm{~Pa})$. The experimental setup is schematically represented in figure 1. A detailed description is given elsewhere $[22,49]$. The top electrode is RF-driven, while the bottom electrode is grounded. The reactor walls are also grounded. The inter-electrode distance is $3.5 \mathrm{~cm}$. The top electrode $(10 \mathrm{~cm})$ is smaller in diameter than the bottom one $(12 \mathrm{~cm})$; it is this which induces a self-bias voltage $\left(\mathrm{V}_{\mathrm{dc}}\right)$ on the top electrode. For the experiments presented in this study, a silver target is placed on the RF-driven electrode. This silver target in combination with the HMDSO precursor is used for the growth of nanocomposite thin films containing silver nanoparticles (from sputtering of the silver target) embedded inside an organosilicon matrix (from plasma polymerization of HMDSO) through hybrid physical vapour deposition (PVD) and plasma enhanced chemical vapour deposition (PECVD) experiments $[49,50]$. The plasma is sustained by a power in the range $5-30 \mathrm{~W}$, resulting in a self-bias voltage between $-265 \mathrm{~V}$ and $-590 \mathrm{~V}$. Variations of approximately $\pm 40 \mathrm{~V}$ around the averaged values of the self-bias voltage are observed during the formation and disappearance cycles of the dust cloud. Given the small populations of sputtered silver atoms or their complete absence over the range of experimental conditions investigated (due to target poisoning in the presence of HMDSO and the low sputtering rates at low RF powers), the contribution of silver species in gas phase reactions can reasonably be ignored. The reactor walls and electrodes were cleaned before each set of experiments.

To ensure homogeneity, the gas mixture is introduced through a ring surrounding the top electrode. Argon is used as a vector gas with a flow of $2.7 \mathrm{sccm}$ (standard cubic centimeters per minute at standard temperature and pressure), and its partial pressure is measured to $5.33 \mathrm{~Pa}$ using a MKS Baratron gauge. HMDSO serves as a precursor gas for the dust generation. It is injected by pulses with a frequency fixed at $0.2 \mathrm{~Hz}$. The HMDSO duty cycle is varied between $30 \%$ and $90 \%$, depending on the pulse duration. The HMDSO flow is adjusted by a mass flow controller (OMICRON) switched by a pulse generator (AGILENT). The maximum HMDSO flow is 0.4 $\mathrm{sccm}$; this corresponds to a continuous injection. The gases are mixed in a buffer chamber before being introduced into the plasma. The total pressure in the discharge slightly oscillates due to the pulsed injection of HMDSO, rising up to $6.13 \mathrm{~Pa}$ for the highest HMDSO duty cycle. To better understand the importance of HMDSO injection on the plasma properties, all experiments are performed following the same protocol. A nominally pure argon plasma is sustained for a few tens of seconds before HMDSO is injected into the plasma reactor. After two or three generations of dust, HMDSO injection is stopped, and the plasma returns to its initial state. It is worth mentioning that the RF power in this reactor is matched using a manual matching box. Impedance matching is performed before HMDSO injection in the nominally pure argon plasma and remains fixed following pulsed injection of HMDSO. No change in the reflected power was observed over the range of experimental conditions investigated, such that the absorbed power (incident minus reflected one) can reasonably be assumed constant throughout the whole experiment.

\subsection{Dynamical processes in RF dusty plasma: multi-scale approach}

In addition to the typical dynamical processes in an RF capacitively coupled discharge, where the applied RF signal, the frequency of electron-atom collisions and the plasma frequencies (both electron- and ion-ones) occur in the $1.0-500 \mathrm{MHz}$ domain (figure 2), the dusty plasma examined in this study involves a number of other processes distributed over a wide range of frequencies: collisions between heavy particles (ions, neutrals and radicals) typically occur in the $10-100 \mathrm{kHz}$ range, the precursor is injected by pulses, usually with a frequency 


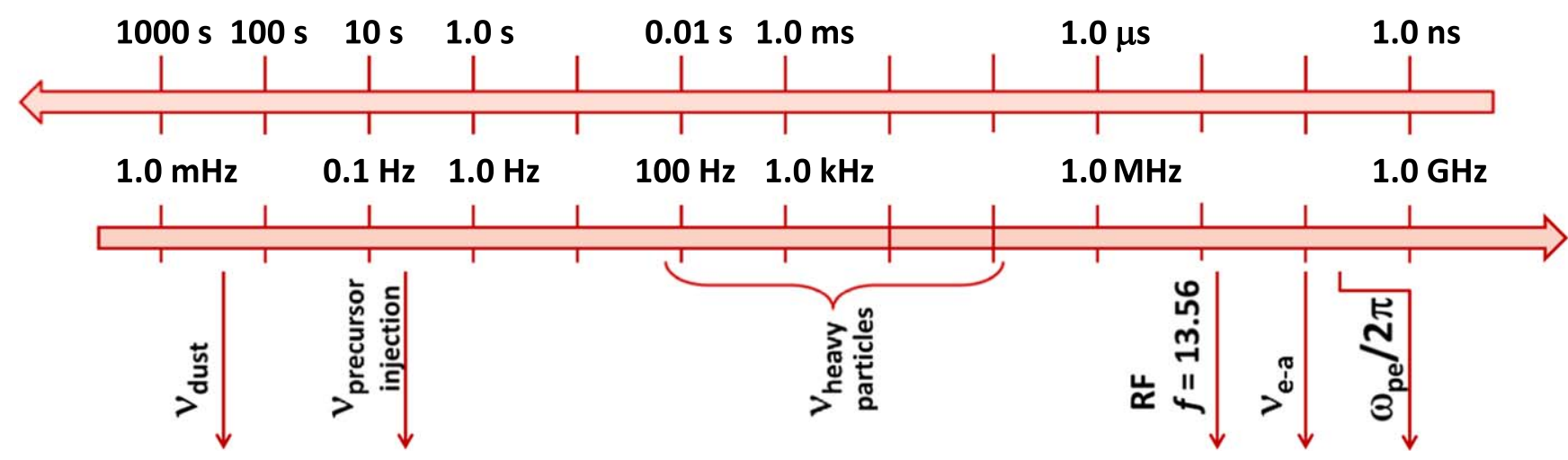

Figure 2. A schematic representation of the time (upper scale) and frequency (lower scale) domains, and the typical dynamical processes in $\mathrm{RF}$ dusty plasmas. The plasma frequencies are calculated for low-pressure plasma at $\mathrm{p}=10 \mathrm{~Pa}, T_{\mathrm{g}}=300 \mathrm{~K}, T_{\mathrm{e}}=2.5 \mathrm{eV}, n_{\mathrm{e}}=10^{9} \mathrm{~cm}^{-3}$.

situated in the $0.1-0.2 \mathrm{~Hz}$ range and the cyclic behaviour of formation/disappearance of successive generations of the dust is typically observed at frequencies of a few $\mathrm{mHz}$. Figure 2 summarizes the main dynamical processes observed in the plasma following the time evolution (upper scale) and frequency domain (lower scale) in RF dusty plasmas sustained at low pressure with pulsed injection of the precursor. Accordingly, this very broad frequency spectrum requires analysis performed at multi-scale to consider the variety of processes occurring in dusty plasma. The multi-scale approach used in this study consists of a combination of time-resolved OES measurements and collisional-radiative modeling to consider different processes in the frequency domain when describing the behaviour of Ar/HMDSO dusty plasma with pulsed injection of HMDSO.

\subsection{Optical diagnostics: a short overview of the processes in the studied Ar/HMDSO dusty plasma}

OES measurements are performed with an AvaSpec 3648 optical spectrometer (Avantes Inc.). An optical fiber is installed to capture the plasma emission (figure 1). It points on a quartz window on the lower half of the discharge between the two electrodes. Since this study involves phenomena occurring at the frequency of HMDSO injection, i.e. $0.2 \mathrm{~Hz}$, the integration time of the detectors in the spectrometer is selected to be small, namely around $500 \mathrm{~ms}$, to acquire an appreciable number of data points over each HMDSO injection cycle.

A Xe-Hg lamp located outside the chamber is used to visualize the dust presence in the plasma. The axes of the lamp and the measuring optical fiber make an angle of $120^{\circ}$ (figure 1). When dust starts growing, the particles act as scattering centers for the light of the lamp. Thus, the recorded signal from $\mathrm{Hg}$-lines corresponds to a scattering intensity and not to an extinction one. This detection method appears very sensitive to follow the formation of successive generations of dust in the plasma. However, it cannot be used to precisely obtain the dust size and density or to map the dust cloud due to the application limit of the Rayleigh's equation [22]. It should be noted that the lamp is not focused, such that it covers most of the plasma volume. Both scattering and OES are recorded at the same spot with the same optical fiber. Both

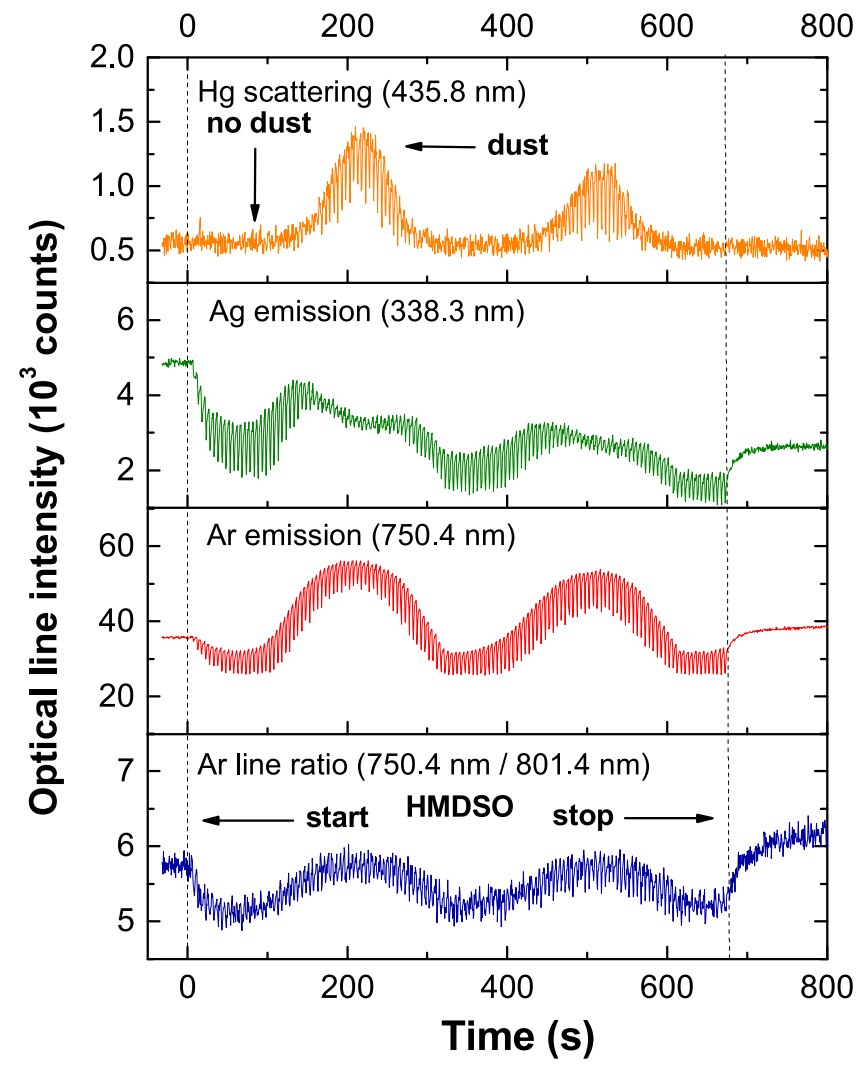

Figure 3. Evolution of the $\mathrm{Hg}$ scattering signal, $\mathrm{Ag}$ emission signal, Ar emission and Ar-line ratio over the time scale of dust cloud formation and disappearance (frequency domain $\mathrm{mHz}$ ) for typical $\mathrm{Ar} / \mathrm{HMDSO}$ conditions: Ar partial pressure $\mathrm{p}_{\mathrm{Ar}}=5.33 \mathrm{~Pa}$; HMDSO duty cycle $=30 \%$; applied power $\mathrm{P}=30 \mathrm{~W}\left(\mathrm{~V}_{\mathrm{dc}}=-590 \mathrm{~V}\right)$.

experiments are thus expected to cover comparable optical paths along the line of sight.

The observed optical spectra offer direct information on the processes at play in the plasma during the dust formation/ disappearance cycle at multi-scale. To give an overview of the processes in the studied Ar/HMDSO dusty plasma, we briefly describe here the physical situation before the thorough analysis presented in the next sections. Figure 3 shows the evolution of the principal optical signals $(\mathrm{Hg}, \mathrm{Ag}, \mathrm{Ar}, \mathrm{Ar}-\mathrm{line}$ ratio) for a set of experimental conditions used in this study: 
injection of HMDSO at $0.2 \mathrm{~Hz}$ with the duty cycle fixed at $30 \%$, discharge power of $30 \mathrm{~W}\left(\mathrm{~V}_{\mathrm{dc}}=-590 \mathrm{~V}\right)$, base argon pressure of $5.33 \mathrm{~Pa}$ and total pressure during HMDSO injection of $5.47 \mathrm{~Pa}$. Time $t=0 \mathrm{~s}$ in the figure, as well as in all following figures, corresponds to the moment at which the HMDSO injection starts.

The recorded Hg-light $(\lambda=435.8 \mathrm{~nm})$ in figure 3 corresponds to the scattering intensity from the lamp and is related to the presence of nanoparticles in the plasma. In the current study, this is used to define 'dusty' and 'non-dusty' conditions. 'Dusty' conditions correspond to the times in the experiment where the scattering signal reaches a maximum value. Since the light scattering intensity increases sharply with the number of the particles, such times are linked to the presence of a large number of particles. On the other hand, 'non-dusty' corresponds to the times in the experiments where the scattering signal reaches a minimum value, indicating that the sizes of the particles are below $49 \mathrm{~nm}$ in radius, considering the application limit of the Rayleigh's equation for the wavelength of the reported $\mathrm{Hg}$-line [22]. The transition between the two conditions is gradual. For the set of experimental conditions presented here, the corresponding frequency of the dust formation/disappearance cycle is $3.6 \mathrm{mHz}$. The emission lines recorded on the spectrum are those of argon and silver, with the former due to the vector gas and the latter due to sputtering from the RF-driven electrode. The emission intensity of the $\mathrm{Ag}$-line $(\lambda=338.3 \mathrm{~nm})$ is primarily dependent on the sputtering rate of silver atoms, which is related to the applied discharge power, and the coverage of the target surface by reactive species from the HMDSO fragmentation and recombination reactions. The slow decrease in the Ag signal through successive dust generations indicates a progressive poisoning of the target for these experimental conditions. For fixed plasma conditions (power, pressure, etc), the plasma emission of an argon discharge depends on the electron density and mean electron energy, which are involved in the excitation of the Ar atoms. The oscillation of the Ar signal $(\lambda=750.4 \mathrm{~nm})$ means that there is a change either in the electron temperature or electron density, or both, following HMDSO injection and formation of the dust cloud. To quickly gain more insight, the similar evolution of the ratio of two Ar-lines, shown in the last panel of figure 3, emphasizes a possible variation of the electron temperature.

The fast oscillations that can be observed in figure 3 are not noise signals. In fact, they show a second cyclic behavior of the plasma at a higher frequency, namely at the pulsed injection of HMDSO with a frequency of $0.2 \mathrm{~Hz}$. Figure 4 shows the evolution of the same four signals as in figure 3 , but at this higher frequency. The grey areas in the figure represent the time during which HMDSO is injected in the plasma. As mentioned earlier, all experiments start with pure Ar discharge (time preceding the indicated $t=0 \mathrm{~s}$ on the time axis). The recorded intensity of $\mathrm{Hg}$-lines is at the noise level. As observed earlier, the HMDSO injection starts at $t=0 \mathrm{~s}$ and the first time interval on figure 4 , before the break on the time axis, corresponds to the very first $10 \mathrm{~s}$, where no evolution of the spectrum is neither expected nor detected. Later, at the stage before dust appearance $(105-120 \mathrm{~s}$ on the time scale), there is still no $\mathrm{Hg}$ scattering. In the presence of dust

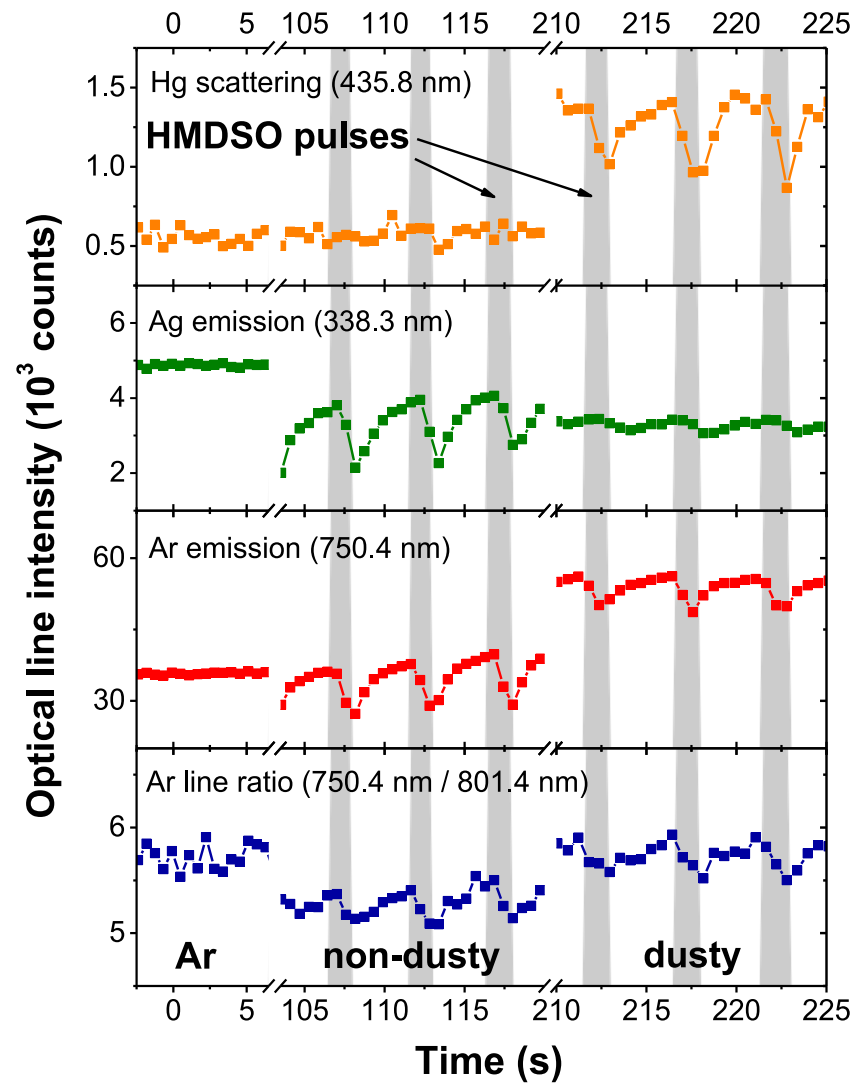

Figure 4. Evolution of the $\mathrm{Hg}$ scattering signal, $\mathrm{Ag}$ emission signal, Ar emission and Ar-line ratio over the time scale of HMDSO injection pulses (frequency of $0.2 \mathrm{~Hz}$ ) in pure argon discharge (left), $\mathrm{Ar} / \mathrm{HMDSO}$ non-dusty (middle) and dusty conditions (right). The grey areas represent the HMDSO injection time-on for typical Ar/HMDSO conditions: Ar partial pressure $\mathrm{p}_{\mathrm{Ar}}=5.33 \mathrm{~Pa}$; HMDSO duty cycle $=30 \%$; applied power $\mathrm{P}=30 \mathrm{~W}\left(\mathrm{~V}_{\mathrm{dc}}=-590 \mathrm{~V}\right)$.

(210-225 s on the time scale) the scattering of the Hg-line shows a quick drop during the HMDSO injection pulse, suggesting that either the dust density or their size decreases instantly after HMDSO introduction. It is reasonable to expect that the size of the nanoparticles does not vary significantly at the HMDSO injection frequency $(0.2 \mathrm{~Hz})$, since the frequency of appearance of successive generations of nanoparticles is much lower (in the $\mathrm{mHz}$ range). Hence, it is suspected that the density of the dust cloud in the scattering area changes. As discussed in a previous work, this observation cannot be explained by blowing effects related to the reactive gas drag at the opening of the flow controller [22]. The HMDSO flow rate in this case is small $(0.12 \mathrm{sccm}$ for a HMDSO duty cycle of $30 \%$ ) as well as the total pressure variation (only $0.13 \mathrm{~Pa}$ ). This effect is most certainly due to a change in the balance of forces confining the cloud in the plasma region. For example, a change in the density of charged species in the plasma due to their attachment on the surface of nanoparticles could induces a variation in the amplitude of the inward electrostatic force, which is known to be of critical importance [13]. The $\mathrm{Ag}$ emission signal also decreases during the injection timeon, but only in non-dusty conditions. This is easily explained by the fact that reactive species originating from HMDSO 
dissociation in the plasma partially cover the silver target, diminishing the sputtering yield of metal. When dust grows in the plasma, it consumes an important part of the HMDSO fragments, which reduces the impact on the target poisoning. It is important to note that the Ag emission signal is significant only for very low HMDSO duty cycles $(\leqslant 30 \%)$. Otherwise it is lost almost immediately after the beginning of HMDSO injection. Finally, the emission of the Ar-lines and their line ratio both decrease during the injection pulses in non-dusty and dusty conditions, indicating that the plasma is always instantly sensible to the injection of the reactive agent. It is valuable for the entire Ar spectrum.

The cyclic phenomena presented above have already been observed for the same Ar/HMDSO dusty plasma $[22,23,48]$, and its response was analysed in the $\mathrm{mHz}$ frequency domain using TRG-OES coupled with a collisionalradiative model $[48,51]$. It was shown that both the electron temperature and density have cyclic behaviour, with a frequency corresponding to the dust formation/disappearance cycle. The dust acts as a sink of the electrons due to attachment processes. The decrease in the electron density requires an increase in the electron temperature to maintain the discharge. It was also pointed out that the injection of HMDSO makes the ionization processes in the discharge easier compared to nominally pure argon plasma [23]. However, the collisional-radiative model used in TRG-OES is not adapted to follow significant variations in the plasma chemistry, for example, those occurring at the scale of higher frequencies determined by the pulsed HMDSO injection. To consider changes in the plasma behaviour induced by the pulsed HMDSO injection in an effort to describe the mechanisms at play in this complex plasma in a multi-scale approach, an in fine argon collisional-radiative model is elaborated in this work.

\section{Collisional-radiative modeling of the argon emission lines: consideration of the collisional quenching induced by HMDSO and its fragments}

OES measurements of argon 2p-to-1s transitions (in Paschen's notation) between 700 and $900 \mathrm{~nm}$ are compared to the predictions of a zero-dimensional argon collisional-radiative model [52]. This approach is used to determine the electron temperature (assuming a Maxwellian electron energy distribution function (EEDF) [53]), the electron density, as well as the population of argon metastable and resonant (or radiative) 1s states. Given the very low diffusion coefficient of argon excited states over the range of experimental conditions investigated, transport properties of the excited states can be neglected and a zero-dimensional model can be applied for the analysis. The optical emission measurements are integrated over the line of sight, i.e. they are not radially resolved. Therefore, the plasma properties deduced from the comparison between OES and zerodimensional collisional-radiative modeling correspond to values averaged over the line of sight. Inspired by the work of Donnelly [51], the model solves the particle balance equations of all ten $2 p$ levels. The considered argon energy levels are displayed in

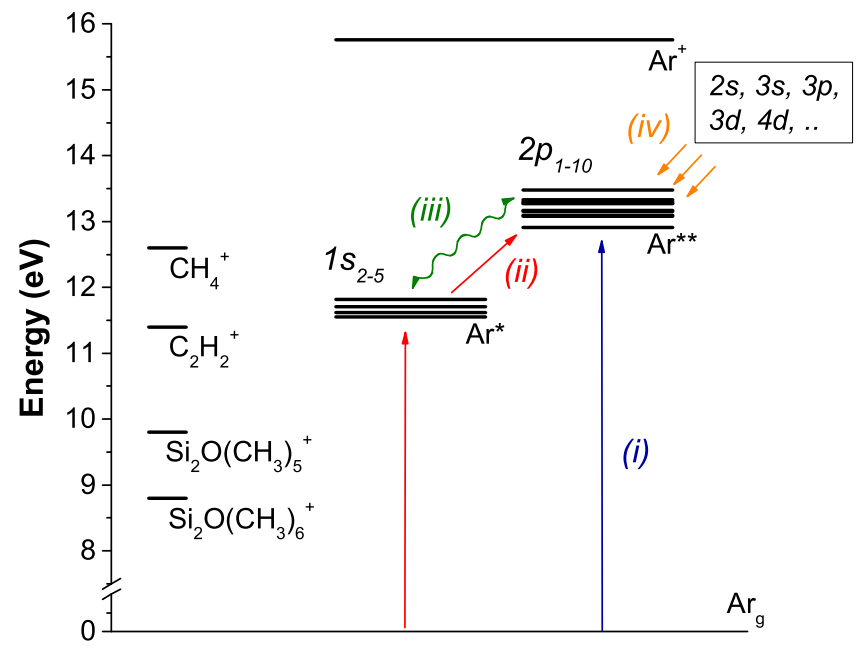

Figure 5. A schematic of argon energy levels with the relevant excitation and de-excitation mechanisms: (i) direct electron-impact excitation of $2 p$ states from the ground state, (ii) stepwise excitation by electron impact, (iii) spontaneous emission and radiation trapping, (iv) cascading from upper levels, (v) direct electron-impact excitation of $1 \mathrm{~s}$ states from the ground state and super-elastic collisions. The ionization energies of HMDSO and its principal fragments are also shown, since these species play an important role in the collisional quenching of argon 1s states.

figure 5, with a scheme of all excitation and de-excitations paths. More precisely, excitation of argon $2 p$ states is assumed to occur by direct electron impact on ground state argon atoms, and by stepwise excitation involving argon 1s states. In contrast to direct excitation that requires electrons from the tail of the EEDF with $13 \mathrm{eV}$ or more, stepwise excitation involves any electron with more than $1.1 \mathrm{eV}$. Over the range of experimental conditions investigated, de-excitation of argon $2 p$ states mostly occurs by spontaneous emission. In contrast to most studies reported in the literature, the model here is refined to consider radiation trapping of argon 2p-to-1s transitions. This is realized by calculating the escape factors of each transition using Mewe's formula [54]. Such effects become significant in plasmas characterized by relatively high populations of argon 1s states, and when the optical path of photons originating from argon 2p-to-1s transitions becomes smaller than the typical dimension of the plasma along the line of sight. In this experiment, radiation trapping effects are found to be important. This collisional-radiative model is more precise than the corona model typically used in dusty plasmas [44-47] that only considers electron-impact excitation from the ground state, and thus fully neglects the role of atoms in metastable or resonant states.

To adequately describe stepwise excitation by electron impact and radiation trapping, the model also solves balance equations for the population of argon 1s states. As described by Donnelly [51] and recalled in figure 5, these states are populated by direct electron impact on the ground state and by spontaneous emission of $2 p$ levels. Depopulation of $1 \mathrm{~s}$ states occurs through stepwise excitation, super-elastic collisions and quenching. Mixing of argon $1 \mathrm{~s}$ and $2 \mathrm{p}$ states by electron collisions is also considered $[55,56]$. The full set of cross sections used in this study were reviewed by Donnelly 


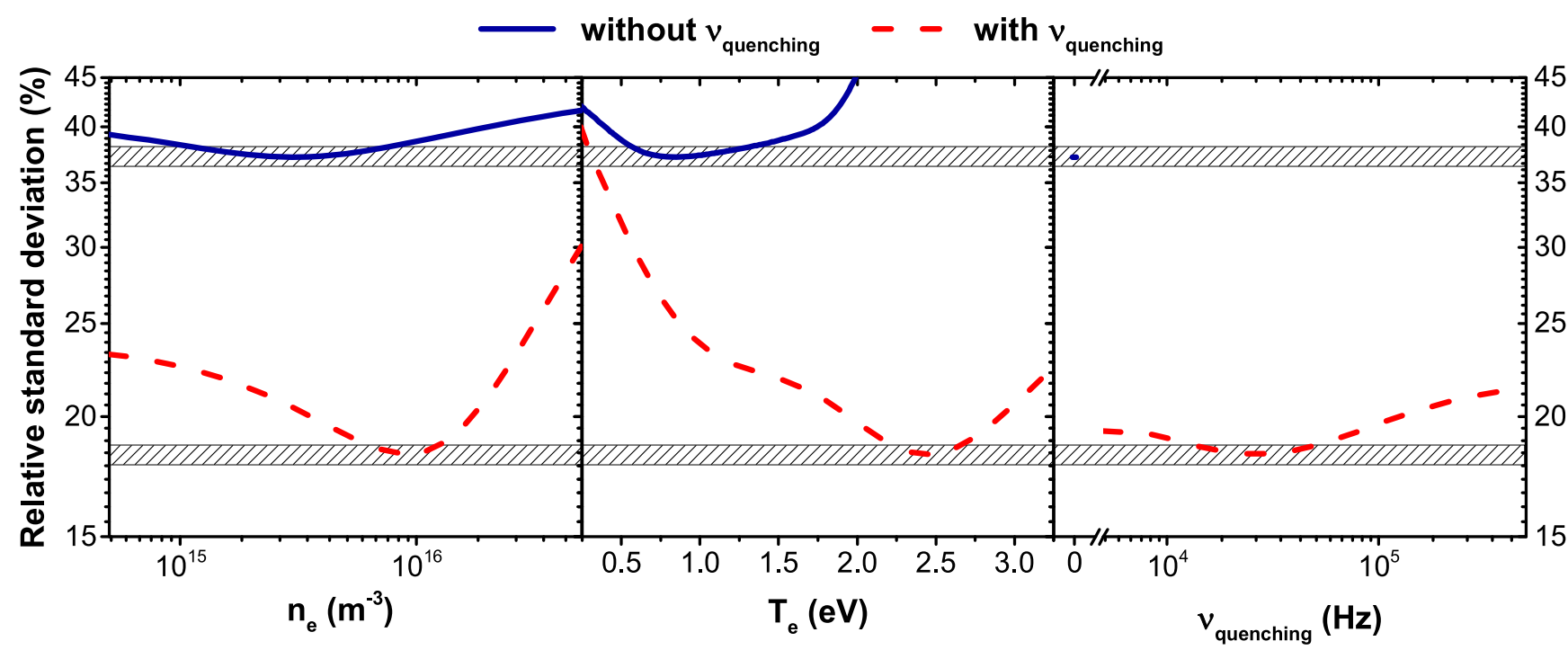

Figure 6. Evolution of the RSD with the electron temperature, electron density and collisional-quenching frequency of argon $1 \mathrm{~s}$ states in typical Ar/HMDSO conditions: Ar partial pressure $\mathrm{p}_{\mathrm{Ar}}=5.33 \mathrm{~Pa}$; HMDSO duty cycle $=30 \%$; applied power $\mathrm{P}=30 \mathrm{~W}\left(\mathrm{~V}_{\mathrm{dc}}=-590 \mathrm{~V}\right)$.

[51]. In the case of electron-impact excitation reactions, these cross sections consider cascading effects such that there is no need to include the populations of argon upper levels in the collisional-radiative model.

In argon plasmas in the presence of precursors such as HMDSO, many species are expected to play important roles in the quenching of argon 1s states, and thus on the population-depopulation dynamics of argon $2 p$ states. For example, over the range of experimental conditions investigated, the dissociation degree of HMDSO reaches 95\% [23]. In such plasmas, a large number of compounds generated in the gas phase or at the chamber walls, including acetylene $\left(\mathrm{C}_{2} \mathrm{H}_{2}\right)$ and methane $\left(\mathrm{CH}_{4}\right)$ [23, 57], participate in the quenching of metastable and resonant argon atoms [58-60]. More specifically, if the excitation or ionization energy thresholds of a molecule present in the plasma are below the energy of argon $1 \mathrm{~s}$ states, Penning excitation or ionization of this molecule will be promoted. Ionization energy thresholds of a few species related to HMDSO-based plasmas are presented in figure 5 [61, 62]. As can be seen, $\mathrm{Si}_{2} \mathrm{O}\left(\mathrm{CH}_{3}\right)_{6}$ (HMDSO), $\mathrm{Si}_{2} \mathrm{O}\left(\mathrm{CH}_{3}\right)_{5}$ (HMDSO-15, i.e. HMDSO without one methyl group) and $\mathrm{C}_{2} \mathrm{H}_{2}$ (acetylene) can all contribute to the quenching of argon $1 \mathrm{~s}$ states by Penning ionization, with a large contribution of $\mathrm{C}_{2} \mathrm{H}_{2}$ considering the efficiency of this process due to the small difference between the excitation and ionization energies of the two species. $\mathrm{CH}_{4}$ is the only molecule here with an ionization energy threshold above the energy of the argon 1s levels. Nonetheless, this molecule could still induce quenching through dissociation or Penning excitation reactions. While organosilicon products such as $\mathrm{Si}_{2} \mathrm{O}\left(\mathrm{CH}_{3}\right)_{5}$ are directly linked to the HMDSO fragmentation, methane and acetylene are mostly created by heterogeneous surface recombination reactions on the electrodes and chamber walls [63].

In collisional-radiative modeling, quenching processes are generally treated as a quenching frequency involving both the reaction rate and the number density of quenching species. Unfortunately, data on the quenching rate of argon metastable atoms by HMDSO and its related compounds are lacking [64]. Moreover, the population of HMDSO and its related compounds generally strongly varies with the plasma operating conditions. This aspect is expected to be of particular importance in the highly dynamic plasma examined in this study, characterized by multi-scale variations of the plasma response in the frequency domain [23]. Rather than using an approximate and constant quenching frequency for HMDSO and its related compounds as input parameters in the model, the total quenching frequency is treated as an adjustable parameter, together with the electron temperature and electron density.

In this framework, the line emission intensities linked to the population of emitting argon $2 p$ states can be computed over a wide range of electron temperatures, electron densities and quenching frequencies. These quantities can thus readily be determined by comparing the measured emission intensities to those predicted by the model using the electron temperature, electron density and quenching frequency as the only fitting parameters. Results are found by minimising the relative standard deviation (RSD), calculated by dividing the standard deviation of the theoretical-to-experimental line ratios with respect to their mean value [55]. It is worth mentioning that a three-parameter space could a priori reveal more than one combination to fit the experimental data. However, over the range of reasonable electron densities $\left(10^{14}-10^{17} \mathrm{~m}^{-3}\right)$, electron temperatures $(0.1-10 \mathrm{eV})$ and quenching frequencies for metastable argon atoms $\left(10^{3}-10^{6} \mathrm{~s}^{-1}\right)$ for RF argon plasmas at low pressure, only a single minimum (unique solution) was observed for all conditions in the RSD plots.

Figure 6 presents the evolution of the RSD with the three adjustable parameters for a typical spectrum in the $\mathrm{Ar} /$ HMDSO plasma in dusty conditions. First, the RSD is given when varying the electron temperature and electron density 


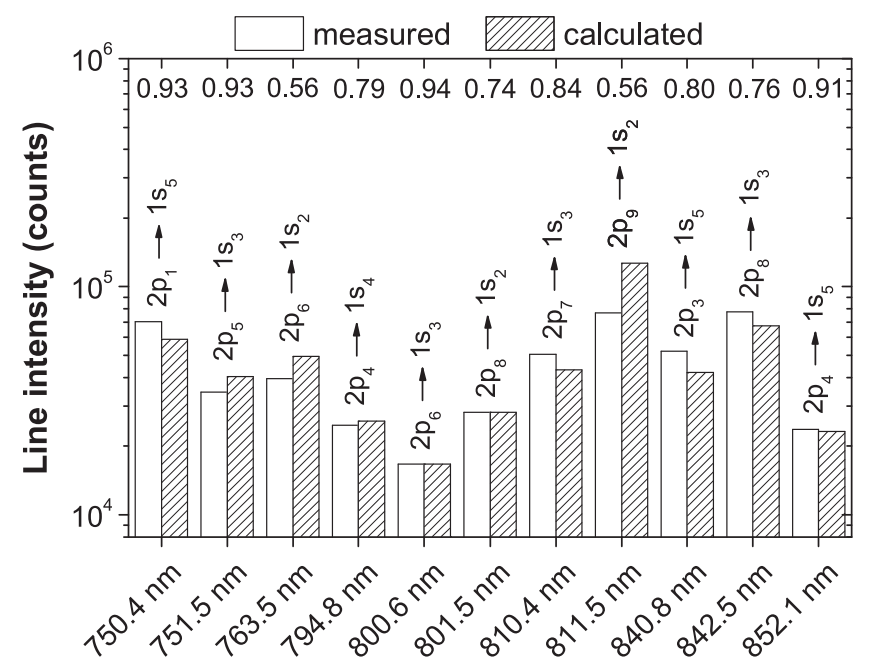

Figure 7. Measured and calculated intensities of the observed argon lines for a typical spectrum in Ar/HMDSO dusty conditions. Their corresponding 2p-to-1s transition and escape factor are also given for typical Ar/HMDSO conditions: Ar partial pressure $\mathrm{p}_{\mathrm{Ar}}=5.33 \mathrm{~Pa}$; HMDSO duty cycle $=30 \%$; applied power $\mathrm{P}=30 \mathrm{~W}\left(\mathrm{~V}_{\mathrm{dc}}=\right.$ $-590 \mathrm{~V})$.

without considering any quenching in the model (full curves). A single minimum is observed at $37.2 \%$, with a very low electron temperature, below $1 \mathrm{eV}$. The dashed curves show the results for an adjustable quenching frequency. The RSD then has a much lower minimum, at $18.3 \%$, while the electron temperature and the electron density increase. The RSD shows a single minimum distribution in the third graph, indicating that there is indeed a quenching frequency at which the model optimally matches the measured optical emission spectrum. The value of the quenching frequency varies during the experiment, but it is always located between $10^{4}$ and $10^{5}$ $\mathrm{Hz}$. This concords well with the value of $4.0 \times 10^{4} \mathrm{~Hz}$ found using the collisional-quenching cross section provided by Jauberteau et al [64].

Throughout the experiment, the same set of spectral lines needs to be selected for each recorded spectrum to obtain a proper estimation of the evolution of the plasma parameters. The measured and calculated intensities of the same spectrum used in figure 6 is shown in figure 7, presenting the selection of lines used in this study with the corresponding transition between the energy levels and the escape factor related to radiation trapping. Only radiative de-excitations from $2 \mathrm{p}$ levels down to $1 \mathrm{~s}$ levels with wavelengths between $700 \mathrm{~nm}$ and $900 \mathrm{~nm}$ are measured. Finally, radiation trapping is indeed important, since six out of the followed eleven lines are absorbed by at least $20 \%$ or more.

\section{Results and discussion}

\subsection{Evolution of dusty plasma at multi-scale in the frequency domain}

The argon collisional-radiative model developed in this work to extract the plasma parameters after treatment of the recorded OES spectra of Ar/HMDSO dusty plasma has the

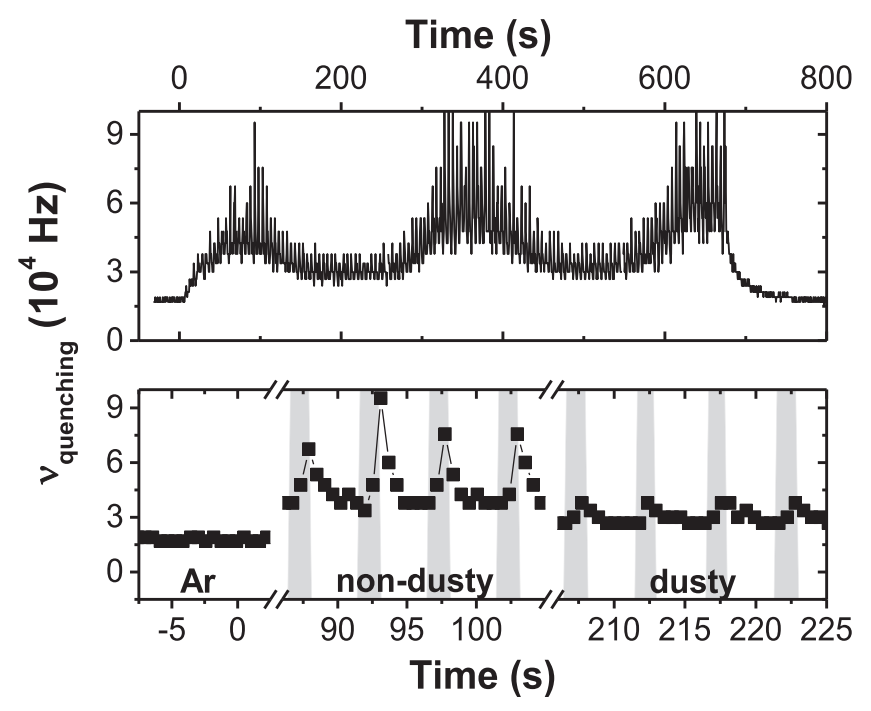

Figure 8. Multi-scale temporal development of the collisionalquenching frequency during dust formation/disappearance cycles (upper panel) and HMDSO injection pulses (lower panel). The grey areas represent the HMDSO injection time-on. Typical Ar/HMDSO conditions: Ar partial pressure $\mathrm{p}_{\mathrm{Ar}}=5.33 \mathrm{~Pa}$; HMDSO duty cycle $=30 \%$; applied power $\mathrm{P}=30 \mathrm{~W}\left(\mathrm{~V}_{\mathrm{dc}}=-590 \mathrm{~V}\right)$.

main merit of simultaneously tracking processes occurring in dusty plasmas at all frequencies, as presented in section 2.2. This multi-scale approach is achieved by detailed analysis of the electron temperature, electron density and quenching frequency used as fitting parameters in the argon collisionalradiative model. As detailed above, the quenching frequency accounts for the quenching of the argon metastable and resonant atoms by HMDSO and its related compounds.

4.1.1. Quenching frequency and Ar-metastable density. The temporal evolution of quenching frequency is shown in figure 8 . The variation of $\nu_{\text {quenching }}$ is at two frequencies: (i) the very low frequency of $3.6 \mathrm{mHz}$ corresponding to the cyclic formation of dust in the plasma (figure 8, upper panel), and (ii) the HMDSO injection frequency of $0.2 \mathrm{~Hz}$ (figure 8, lower panel). As described above, HMDSO injection starts at $t=0 \mathrm{~s}$, as displayed in figure 8. At this moment, a drastic increase in the quenching of argon metastable atoms is observed; a feature obviously linked to the injection of HMDSO and the appearance of its main compounds $\left(\mathrm{C}_{2} \mathrm{H}_{2}\right.$, $\mathrm{CH}_{4}$ and $\mathrm{Si}_{2} \mathrm{O}\left(\mathrm{CH}_{3}\right)_{5}$ (HMDSO-15)) [23, 57]. On average, over the very low-frequency variation (figure 8, upper panel), the quenching frequency first increases to reach a maximum, and then decreases to a plateau. However, values remain higher by about a factor of two with respect to those observed in the nominally pure argon discharge. The maximum and following decrease of $\nu_{\text {quenching }}$ occurs when dust starts to grow in the plasma. HMDSO and its plasma-generated compounds see their density decrease with dust formation and growth, as previously reported through plasma sampling mass spectrometry measurements [23]. Smaller fragments, resulting from the $\mathrm{C}_{2} \mathrm{H}_{2}$ dissociation, are not efficient for quenching argon metastable atoms [60]. Thus, the total quenching frequency decreases with dust growth, but increases again 


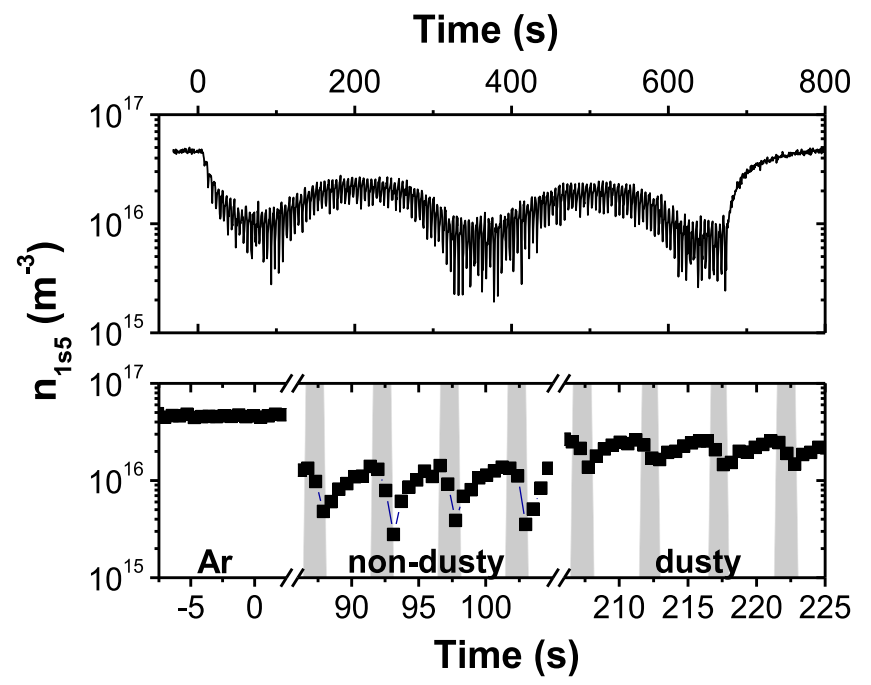

Figure 9. Multi-scale temporal development of the $1 \mathrm{~s}_{5}$ metastable atom density during dust formation/disappearance cycles (upper panel) and HMDSO injection pulses (lower panel). The grey areas represent the HMDSO injection time-on. Typical Ar/HMDSO conditions: Ar partial pressure $\mathrm{p}_{\mathrm{Ar}}=5.33 \mathrm{~Pa}$; HMDSO duty cycle $=30 \%$; applied power $\mathrm{P}=30 \mathrm{~W}\left(\mathrm{~V}_{\mathrm{dc}}=-590 \mathrm{~V}\right)$.

when the dust cloud subsequently disappears from the plasma. This cyclic behaviour continues until the injection of HMDSO is stopped after two complete cycles of formation and disappearance of the dust cloud. The quenching frequency then decreases back to its initial value in the nominally pure argon discharge.

The lower part of figure 8 shows the evolution of the quenching frequency during the pulses of injection of HMDSO, i.e. at the higher frequency of $0.2 \mathrm{~Hz}$. The grey areas represent the $1.5 \mathrm{~s}$ injection time of HMDSO over the $5 \mathrm{~s}$ pulses. It clearly appears that the injection of HMDSO instantly induces quenching of argon metastable atoms. This is true for both dusty and non-dusty conditions, but the effect is reduced by dust presence due to consumption of HMDSO and its related compounds, mainly the $\mathrm{C}_{2} \mathrm{H}_{2}$. Quenching slowly decreases during the HMDSO time-off because of the residence time of HMDSO and its related compounds in the plasma. It never decreases back to its initial value $(t=0 \mathrm{~s})$, meaning that HMDSO fragments remain present, even during the HMDSO time-off, and that nanoparticles do not consume all of the HMDSO fragments.

The populations of metastable and resonant argon atoms are evidently dependent on the variation in the quenching frequency. It is found that once the HMDSO is injected, the collisional quenching becomes the major loss channel for the argon metastable $1 s_{3,5}$ atoms. Figure 9 shows the temporal evolution of the density of argon metastable atoms in the $1 s_{5}$ excited level. This level is the most populated one out of the four 1s levels, since it has the lowest energy. Its density is higher by an order of magnitude compared to the other $1 \mathrm{~s}$ states. However, all four $1 \mathrm{~s}$ levels show similar trends in this experiment. It is clear from figure 9 that the metastable density is inversely related to the quenching rate. At the very low frequency of dust cyclic behaviour it drops when the

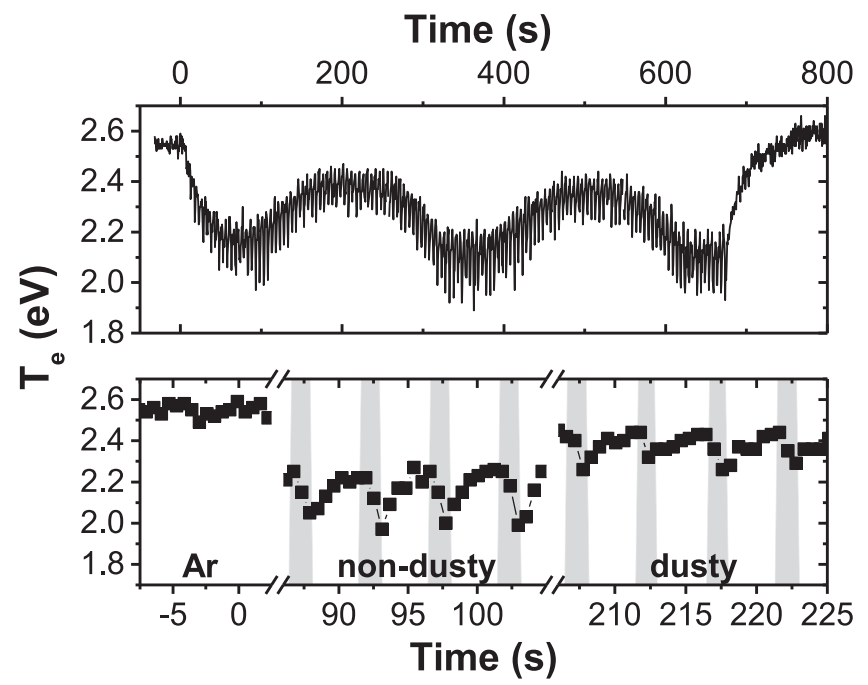

Figure 10. Multi-scale temporal development of the electron temperature during dust formation/disappearance cycles (upper panel) and HMDSO injection pulses (lower panel). The grey areas represent the HMDSO injection time-on. Typical Ar/HMDSO conditions: Ar partial pressure $\mathrm{p}_{\mathrm{Ar}}=5.33 \mathrm{~Pa}$; HMDSO duty cycle $=30 \%$; applied power $\mathrm{P}=30 \mathrm{~W}\left(\mathrm{~V}_{\mathrm{dc}}=-590 \mathrm{~V}\right)$.

HMDSO introduction in the plasma starts, but later on increases when the dust starts growing (figure 9, upper panel) and the quenching is reduced.

The same can be said for the frequency of pulsed injection of HMDSO (figure 9, lower panel). Indeed, the metastable density decreases during the HMDSO injection time-on and goes back up during the time-off, both in dusty and non-dusty conditions. The variations in the metastable density here are significant; the density of atoms in the $1 \mathrm{~s}_{5}$ state decreases by a factor of more than ten. This means that the contribution to stepwise excitation by electron impact of the $2 p$ states and radiation trapping of $2 p$-to- 1 s transitions are compromised by HMDSO. This obviously has a strong impact on the electron density, and consequently on the plasma maintenance.

4.1.2. Electron temperature and electron density. One way to obtain information about the impact of HMDSO introduction and the growth of nanoparticles in plasma is to look at the variations in the main plasma parameters that characterize the electron population, i.e. the electron temperature, $T_{\mathrm{e}}$, and electron density, $n_{\mathrm{e}}$. Figures 10 and 11 present the results obtained from the comparison between measured and simulated OES spectra for the evolution of electron temperature and electron density over the dust cycles. The main observation is that the value of $T_{\mathrm{e}}$ increases when dust appears in the plasma and then decreases during dust disappearance, while $n_{\mathrm{e}}$ shows the opposite trend. This is consistent with previously reported results [48].

Immediately after HMDSO injection, $T_{\mathrm{e}}$ values drop by about $0.5 \mathrm{eV}$ (figure 10, upper panel), while $n_{\mathrm{e}}$ values increase. This phenomenon is due to an addition in the ionization sources in the plasma when transitioning from nominally pure Ar plasma to an Ar/HMDSO plasma (before dust formation, for times up to $t=70 \mathrm{~s}$ ). One of the species 


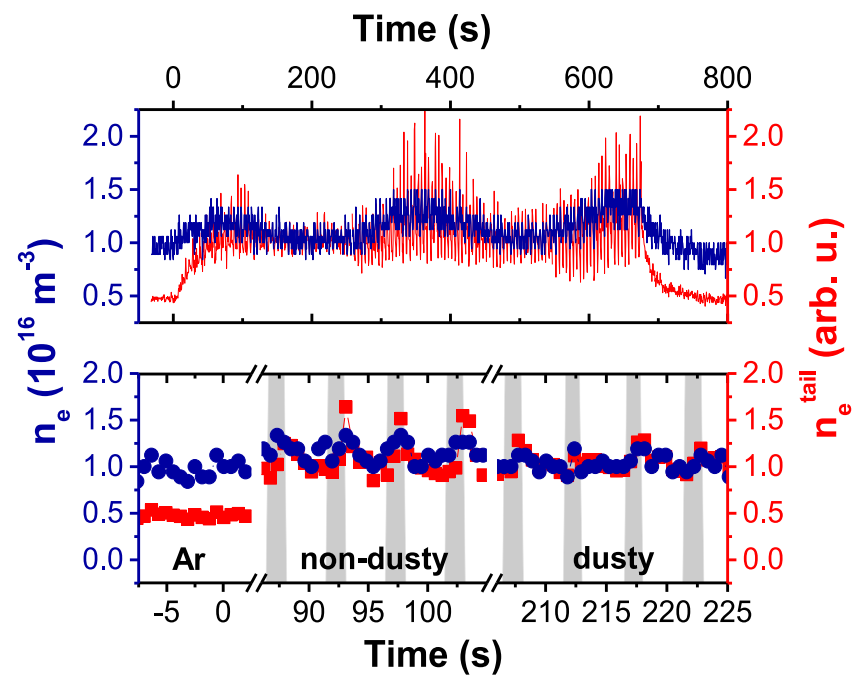

Figure 11. Multi-scale temporal development of the electron density during dust formation/disappearance cycles (upper panel) and HMDSO injection pulses (lower panel). The grey areas represent the HMDSO injection time-on. Typical Ar/HMDSO conditions: Ar partial pressure $\mathrm{p}_{\mathrm{Ar}}=5.33 \mathrm{~Pa}$; HMDSO duty cycle $=30 \%$; applied power $\mathrm{P}=30 \mathrm{~W}\left(\mathrm{~V}_{\mathrm{dc}}=-590 \mathrm{~V}\right)$.

generated after the HMDSO fragmentation is $\mathrm{Si}_{2} \mathrm{O}\left(\mathrm{CH}_{3}\right)_{5}(\mathrm{~m} /$ $\mathrm{z}=147$, HMDSO-15). It is obtained by detachment of one methyl group from the former HMDSO molecule. The process is usually dissociative ionization leading to dominant production of the neutral radical $\mathrm{CH}_{3}{ }^{\circ}$ and $\mathrm{Si}_{2} \mathrm{O}\left(\mathrm{CH}_{3}\right)_{5}{ }^{+}$. The ionization threshold of $\mathrm{Si}_{2} \mathrm{O}\left(\mathrm{CH}_{3}\right)_{5}$ is $9.6 \mathrm{eV}$ [61, 62], much lower than the first ionization energy of argon $(15.76 \mathrm{eV})$, and even below the excitation threshold for the $1 \mathrm{~s}_{5}$ metastable state $(11.55 \mathrm{eV})$ (figure 5). It represents over $90 \%$ of the total ionization cross section of HMDSO products at energies below $25 \mathrm{eV}$ [61]. Furthermore, Penning ionization of the HMDSO fragments (figure 5), mainly $\mathrm{C}_{2} \mathrm{H}_{2}$, during collisional quenching of argon metastable atoms contributes largely to the creation of charged particles in the plasma. The efficiency of this reaction is quite high. As observed in figures 8 and 9, the quenching frequency increases three times after HMDSO injection, while the $1 \mathrm{~s}_{5}$ argon metastable atom density decreases by a factor of five. These two mechanisms, dissociative ionization of HMDSO and Penning ionization of the HMDSO fragments, thus become additional sources for charged particle creation in the plasma leading to a requirement for less energy for the plasma maintenance, i.e. to an important decrease in $T_{\mathrm{e}}$, and therefore, to an increase in the electron density at fixed absorbed power (figures 10 and 11). As a consequence of the decreased electron temperature, the excitation of Ar atoms is reduced, inducing the observed decrease in the argon emission intensity in figure 3, directly after the HMDSO injection.

When nanoparticles grow in the plasma, they collect charges and, most noticeably, they act as an important sink for electrons. Processes behind the charging of nanoparticles levitating in a plasma vary with the size of the particles; protoparticles (less than $2 \mathrm{~nm}$ in size) are formed by negative ions [13], while larger particles that possess a negative sheath at their surface acquire ions and electrons in ways usually understood within the framework of the orbital-motion-limited theory $[65,66]$. The fact that their mean charge increases with their size remains fairly true at all sizes $[16,67]$. This additional loss channel for the electrons was reported to induce changes in the electron temperature [47]. Evidently, the electron density also decreases. The electron loss depends on the size and density of the dust cloud, and on the former electron density, all of which depend on the experimental conditions. In the current experiment, since the density of HMDSO compounds decreases when the nanoparticles grow in the Ar/HMDSO plasma (see figure 7), the efficiency of Penning ionization, which tends to lower $T_{\mathrm{e}}$, also decreases. In response to the dust formation in the plasma (70 $\mathrm{s}<t<210 \mathrm{~s}$ ), the electron temperature increases (figure 10, upper panel), while the electron density decreases (figure 11, upper panel). Each cycle of the dust dynamics is closed by disappearance of the bigger nanoparticles leading to a progressive decrease in the electron temperature and an increase in the electron density. It should also be noted that when the injection of HMDSO stops, at around $t=680 \mathrm{~s}$, the electron temperature and density slowly get back to their initial values, corresponding to a nominally pure argon plasma.

The lower panel in figure 10 shows the evolution of the electron temperature at the frequency of pulsed injection of HMDSO. It follows the same behaviour as the quenching frequency. The electron temperature instantly decreases when the HMDSO is injected in the plasma (the grey areas), and quickly goes up during the injection time-off. This cyclic behaviour of $T_{\mathrm{e}}$ at the HMDSO pulse frequency is always observed throughout the experiment, even with the presence of nanoparticles inside the plasma. It is rather related to the enhanced ionization processes in the plasma by the injection of HMDSO, in particular to the Penning ionization of HMDSO and its related compounds through collisional quenching of the $1 \mathrm{~s}_{5}$ argon metastable atoms, shown in figure 8 .

Two electron populations are presented in figure 11 . The first is the electron density as a direct output from the collisional-radiative modeling of the recorded OES spectra. It characterizes the entire EEDF, since the stepwise excitation, which involves low-energy electrons, is found to play a significant role in the plasma maintenance. The second, obtained from the ratio between the intensity of an argon line and its excitation rate, characterizes the energy tail of the EEDF. The selected line here, at $750.4 \mathrm{~nm}$, is the most intense one in the observed Ar spectrum of the plasma. It has a very high escape factor and comes from a highly excited level, mostly populated by direct electron-impact excitation from the ground state (the energy difference between the two levels is $13.48 \mathrm{eV}$ ), thus the intensity of this line is effectively proportional to the product of the electron density and the excitation rate. The latter is approximated with the Arrhenius formula, using the electron temperature obtained with the model. Thus, the line intensity can be presented as:

$$
I_{750.4 \mathrm{~nm}} \propto n_{\mathrm{e}}{ }^{\text {tail }} \exp \left(-13.48 / k_{B} T_{\mathrm{e}}\right) .
$$

The overall electron density, $n_{\mathrm{e}}$, is ongoing small variations throughout the experiment due to the different 
channels of gain and loss of electrons mentioned above. However, $n_{\mathrm{e}}{ }^{\text {tail }}$ shows a strong increase directly after the start of the HMDSO injection. Godyak et al [68] have reported that, in low-pressure RF plasmas, the EEDF tends to depart from a single Maxwellian distribution when the electronelectron collision frequency becomes too low. In this case, low- and high-energy electrons are characterized by different electron temperatures and electron densities. However, for plasma densities (integrated over the whole EEDF) around $10^{16} \mathrm{~m}^{-3}$, interactions between electrons start to become significant enough so that the low- and high-energy electrons are being characterized by the same electron temperature, i.e. the EEDF becomes a single Maxwellian. Here, the electron number density obtained from the collisional-radiative model is close to $10^{16} \mathrm{~m}^{-3}$. Accordingly, when the electron density increases following HMDSO introduction, the gap between $n_{\mathrm{e}}$ and $n_{\mathrm{e}}{ }^{\text {tail }}$ diminishes (figure 11, upper panel). After that, the evolutions of both electron densities in dusty and non-dusty conditions are quite similar, until the HMDSO injection stops.

Regarding the evolution at the frequency of HMDSO pulsed injection, $n_{\mathrm{e}}{ }^{\text {tail }}$ most noticeably increases during the HMDSO injection in non-dusty conditions, mainly due to the HMDSO-based compounds. This is, however, subdued in dusty conditions since the HMDSO compounds are partially consumed by the growth of nanoparticles. The overall $n_{\mathrm{e}}$ does not seem to vary significantly at the frequency of pulsed injection HMDSO (figure 11, lower panel).

\subsubsection{Ar/HMDSO dusty plasma behaviour in the frequency domain through fast Fourier transform analysis. To approve} the Ar/HMDSO dusty plasma behaviour in the frequency domain, a fast Fourier transform (FFT) analysis is performed on an argon emission line, the quenching frequency, the electron temperature and the electron density (figure 12). All of these quantities show trends of cyclic behaviour at the same two fundamental frequencies for the Ar/HMDSO dusty plasma, underlined by the grey areas in the figure. Their corresponding harmonics, with frequencies equal to two and three times the fundamental frequency, can also be observed. These second and third harmonics appear with non-negligible contributions because the cyclic behaviour of the followed quantities is more complex than simple sinusoids. The first fundamental frequency, at $3.6 \mathrm{mHz}$, corresponding to a period of $280 \mathrm{~s}$, is related to the long cycle of formation and disappearance of dust in the plasma. The second fundamental frequency is at $0.2 \mathrm{~Hz}$. It corresponds to the pulsed injection of HMDSO over a $5 \mathrm{~s}$ period. It is important to note that the electron density possesses a trend at this same frequency after all, meaning that the HMDSO injection has an immediate effect on $n_{\mathrm{e}}$, although the variation in $n_{\mathrm{e}}$ does not appear to be significant in figure 11 (lower panel).

\subsection{General trends in the dusty plasma behaviour according to the plasma operating conditions}

This section presents the general trends in the cyclic behaviour of Ar/HMDSO dusty plasma when the operating parameters are varied inside the range of conditions in which

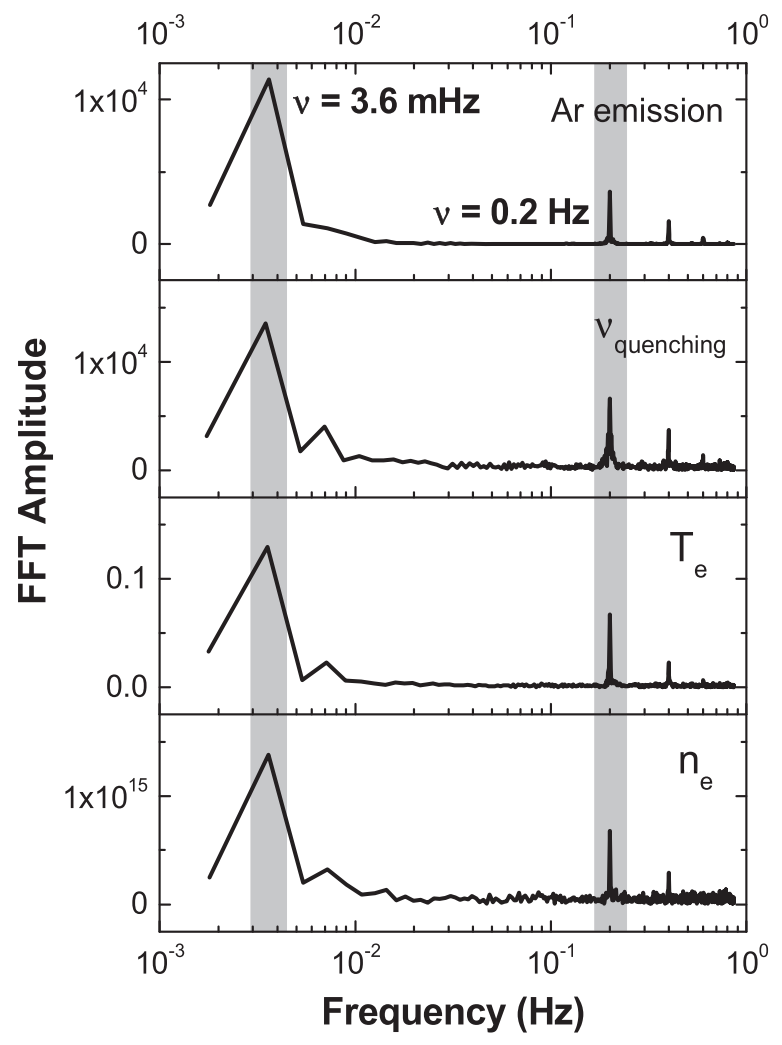

Figure 12. FFT analysis of the time-resolved Ar emission signal $(750.4 \mathrm{~nm})$, the quenching frequency, the electron temperature and the electron density. The fundamental frequencies, corresponding to the cyclic dust formation/disappearance and the HMDSO pulsed injection, are shown in the grey areas. Typical Ar/HMDSO conditions: Ar partial pressure $\mathrm{p}_{\mathrm{Ar}}=5.33 \mathrm{~Pa}$; HMDSO duty cycle $=30 \%$; applied power $\mathrm{P}=30 \mathrm{~W}\left(\mathrm{~V}_{\mathrm{dc}}=-590 \mathrm{~V}\right)$.

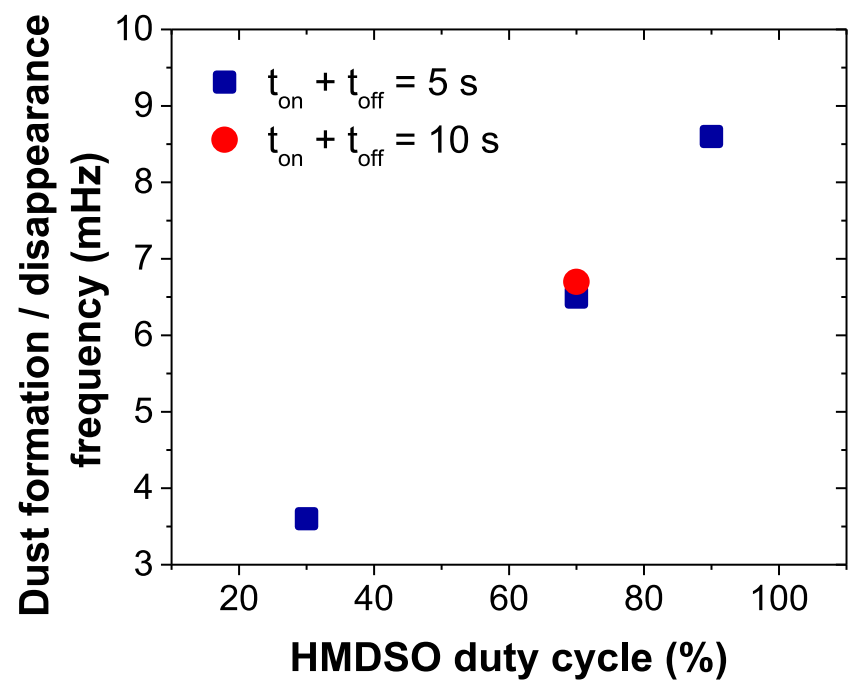

Figure 13. Evolution of the frequency of formation and disappearance of the dust cloud as a function of the HMDSO duty cycle. Ar partial pressure $\mathrm{p}_{\mathrm{Ar}}=5.33 \mathrm{~Pa}$; applied power $\mathrm{P}=30 \mathrm{~W}$ $\left(\mathrm{V}_{\mathrm{dc}}=-590 \mathrm{~V}\right)$.

a dust cloud is observed. It should be noted that dust formation in this experiment is not assured and can be easily avoided. For example, dust formation is not observed for injected power higher than $50 \mathrm{~W}$, or if the HMDSO duty 

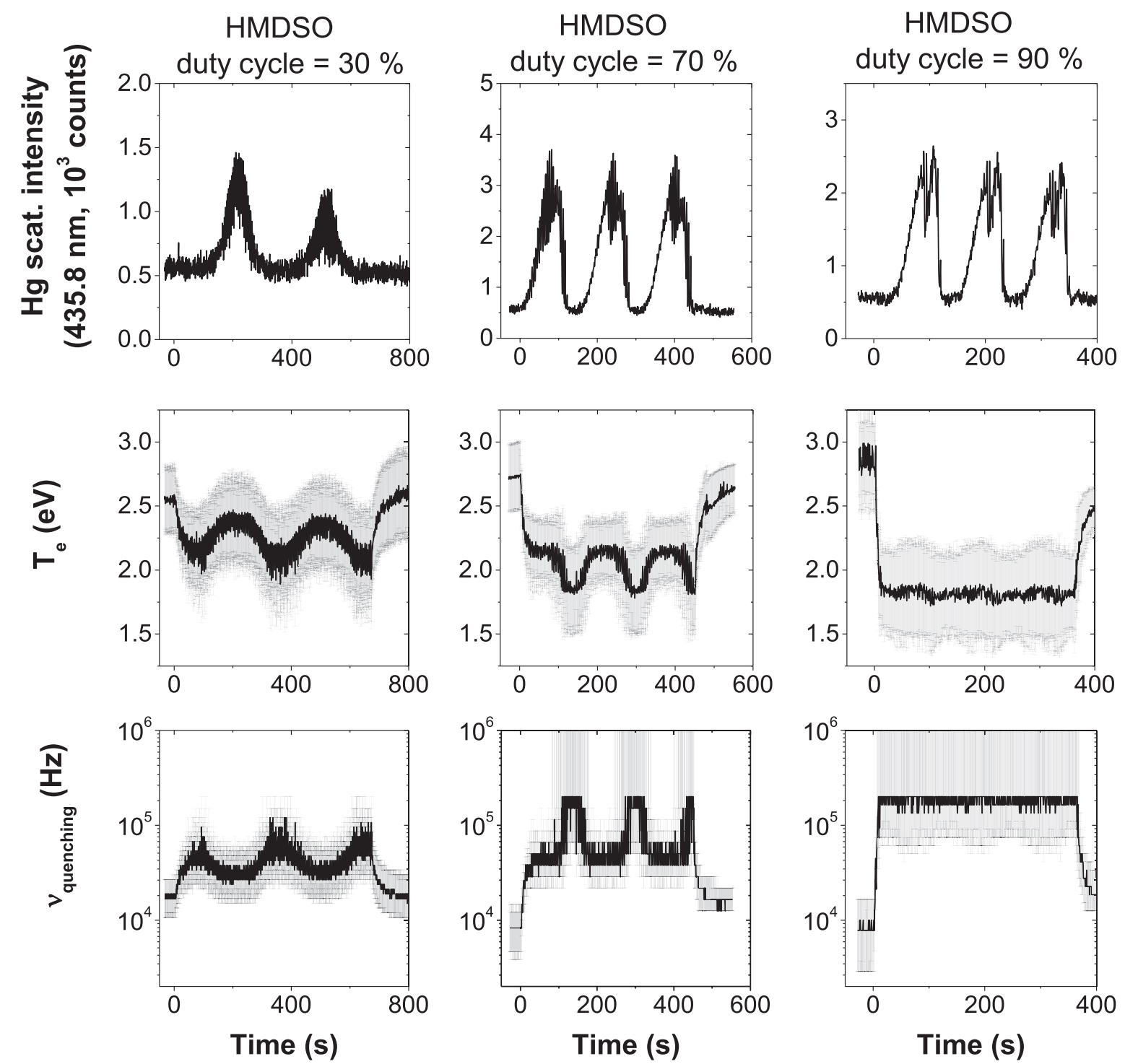

Figure 14. The effect of the HMDSO duty cycle on the temporal development of the Hg scattering intensity at $435.8 \mathrm{~nm}$, the electron temperature and the quenching frequency obtained from the collisional-radiative model of OES spectra recorded in Ar/HMDSO plasma. Error bars are represented in grey. Ar partial pressure $\mathrm{p}_{\mathrm{Ar}}=5.33 \mathrm{~Pa}$; applied power $\mathrm{P}=30 \mathrm{~W}\left(\mathrm{~V}_{\mathrm{dc}}=-590 \mathrm{~V}\right)$.

cycle becomes less than $30 \%$ for an argon partial pressure of $5.33 \mathrm{~Pa}$. Moreover, the regular cyclic formation/disappearance of the dust cloud, without attenuation of the phenomenon, has of yet only been observed for a pulsed injection of HMDSO. Continuous injection of HMDSO leads to the formation of no more than two generations of dust, i.e. two cycles, with strong attenuation of the second cycle, followed by dust disappearance.

To what extent the HMDSO duty cycle is the relevant parameter, rather than the HMDSO pulse injection time-on, for cyclic dust formation in Ar/HMDSO plasma, can be verified by doubling the HMDSO pulse period (figure 13). The dust formation/disappearance cycle remains unchanged for periods of 5 and $10 \mathrm{~s}$ and a duty cycle of $70 \%$. Indeed, practically identical results (not shown) are obtained for the electron temperature, electron density and quenching frequency throughout the whole experiment. The conclusion that can be drawn here is that the processes of nucleation and dust growth are similar for frequencies of pulsed injection of the reactive gas in the $0.1-0.2 \mathrm{~Hz}$ range. The amount of HMDSO injected into the plasma, related to the reactive gas duty cycle, straightforwardly determines the threshold of required reactive species and the time scale at which the dust starts growing.

As shown in figure 13, an increase in the HMDSO duty cycle leads to a linear increase in the dust formation/disappearance frequency. Again, the amount of injected HMDSO into the plasma is the precursor of dust growth, and therefore defines its kinetics. The processes of primary cluster (protoparticles) formation, nucleation and dust growth, coagulation and radical sticking are accelerated when increasing the available amount of reactive species, i.e. the dust kinetics are not limited by the rate constants of the elementary processes. The observed linear dependence underlines the close 
relation between the two frequency scales: the first frquency of the HMDSO pulsed injection and the second frquency of the dust formation/disappearance cycle.

Figure 14 shows the temporal changes in the electron temperature and the quenching frequency obtained from the comparison between measured and simulated OES spectra recorded in Ar/HMDSO plasmas with increasing HMDSO duty cycles. The scattering signal of the $\mathrm{Xe}-\mathrm{Hg}$ lamp is also given as a reference for the presence of successive generations of dust.

First, looking at the time scales, the influence of the HMDSO duty cycle on the frequency of dust formation/ disappearance discussed above is obvious: the entire process of dust formation is accelerated for higher HMDSO duty cycles. Furthermore, the influence of HMDSO and its compounds on the formerly pure Ar plasma $(t=0 \mathrm{~s})$ and $\mathrm{Ar} /$ HMDSO plasma in non-dusty conditions ( $\mathrm{Hg}$ scattering intensity is close to zero) always translates to a decrease in $T_{\mathrm{e}}$ and an increase in $\nu_{\text {quenching. }}$. The reason behind this is mostly the increased ionization due to Penning ionization of HMDSO and its compounds; this can be confirmed by the increase in $\nu_{\text {quenching }}$ obtained from the comparison between OES spectra and the predictions of the collisional-radiative model. For Ar/HMDSO plasma in dusty conditions, the consumption of HMDSO and its compounds for dust formation reduces the $\nu_{\text {quenching }}$ and consequently the Penning ionization, which in combination with the increased losses of electrons due to attachment and screening of dust leads to an increase in electron temperature to compensate the loss of charges.

An increase in the HMDSO duty cycle leads to a progressive decrease in the mean value of $T_{\mathrm{e}}$, around which the electron temperature oscillates during dust cyclic formation. However, the $T_{\mathrm{e}}$ decrease is limited to $T_{\mathrm{e}}=1.8 \mathrm{eV}$ as the quenching frequency approaches $2 \times 10^{5} \mathrm{~Hz}$. When the amount of injected HMDSO and the plasma-generated compounds become very high $(0.36 \mathrm{sccm}$ for a HMDSO duty cycle of $90 \%$ ), the upper limit of $\nu_{\text {quenching }}$ is attained, and the quenching of argon metastable atoms remains saturated. At that point, the periodicity of the main plasma parameters, $T_{\mathrm{e}}$ and $n_{\mathrm{e}}$, in time is lost and the plasma maintenance changes. As long as the saturation regime is maintained, the electron temperature remains low, even in dusty conditions where a portion of reactive fragments is consumed. From the figure, it seems that as long as $\nu_{\text {quenching }}$ is in saturation, $T_{\mathrm{e}}$ is kept below $1.9 \mathrm{eV}$. Due to the saturated quenching of the Armetastable atoms, continuous injection (not shown) of HMDSO leads to the loss of the cyclic phenomena of dust formation/disappearance, as observed experimentally.

Figure 14 shows that the upper limit of $\nu_{\text {quenching }}$ of $2 \times 10^{5} \mathrm{~Hz}$, without saturation of the quenching process, is reached starting in non-dusty conditions at an HMDSO duty cycle of $70 \%$. From a simulation point of view, the RSD is stable past $2 \times 10^{5} \mathrm{~Hz}$, hence the unlimited error bars go above this value. In this regime, the quenching process is simply so important that the metastable argon atoms become insignificant, and thus stepwise excitation reactions play a negligible role in the excitation kinetics of the argon $2 p$ states.
Evidently, a sufficient amount of HMDSO, through the duty cycle, has to be introduced so that the threshold density of protoparticles is obtained and the following stages of dust formation, i.e. nucleation and dust growth, coagulation and radical sticking, occur. Another operating parameter that influences the dust formation is the applied power. For the same total gas pressure and gas mixture, increasing the applied power leads to an increase in the electron density, resulting in a higher fragmentation degree of HMDSO. Subsequently, the processes of dust formation and growth are modified. The influence of the applied power on the temporal evolution of $T_{\mathrm{e}}$ and $\nu_{\text {quenching }}$ is shown in figure 15 for $\mathrm{Ar} /$ HMDSO plasma with an HMDSO duty cycle of $70 \%$. First, it clearly appears that increasing the applied power does not strongly influence the frequency of the dust formation/disappearance cycle.

The applied power needed to create the plasma, however, has a strong effect on the values of the electron temperature and quenching frequency, as obtained from the OES measurements in combination with the collisional-radiative model. In fact, their temporal development throughout the experiment changes in an opposite manner with power as it does with the HMDSO duty cycle. Saturation in the quenching of argon metastable atoms is achieved for $\mathrm{P}=5$ W leading to a stable electron temperature at around $1.8 \mathrm{eV}$, without any oscillations during the dust formation/disappearance cycle. For such a high HMDSO duty cycle (70\%) at low applied powers, the saturation regime of quenching frequency is reached throughout the experiment, such as with a duty cycle of $90 \%$ for higher applied power. When the applied power increases, $\nu_{\text {quenching }}$ slowly starts to decrease, in dusty conditions, meaning that the gain of electrons through the ionization of argon atoms increases and the consumption of HMDSO fragments during dust formation and growth becomes significant.

\section{Conclusion}

We have performed a multi-scale investigation in the frequency domain of an Ar/HMDSO dusty plasma with pulsed injection of HMDSO. The two fundamental frequencies appearing in this complex plasma, namely the very low frequency of dust formation/disappearance cycle and the low frequency of pulsed injection of HMDSO, are simultaneously followed. A combination of time-resolved OES measurements and collisional-radiative modeling was used to study the phenomena occurring during cyclic formation/ disappearance of the dust cloud. The presence of HMDSO in the argon plasma was considered in the model through the quenching of the argon metastable states via HMDSO and its compounds; most efficiently by acetylene $\left(\mathrm{C}_{2} \mathrm{H}_{2}\right)$ and HMDSO-15 $\left(\mathrm{Si}_{2} \mathrm{O}\left(\mathrm{CH}_{3}\right)_{5}\right)$, and to some extent by methane $\left(\mathrm{CH}_{4}\right)$. The kinetics of dust formation in Ar/HMDSO plasmas were described on the basis of the cyclic evolution of the electron temperature, electron density and quenching frequency obtained from the comparison between measured and simulated OES spectra. It was found that the Penning 

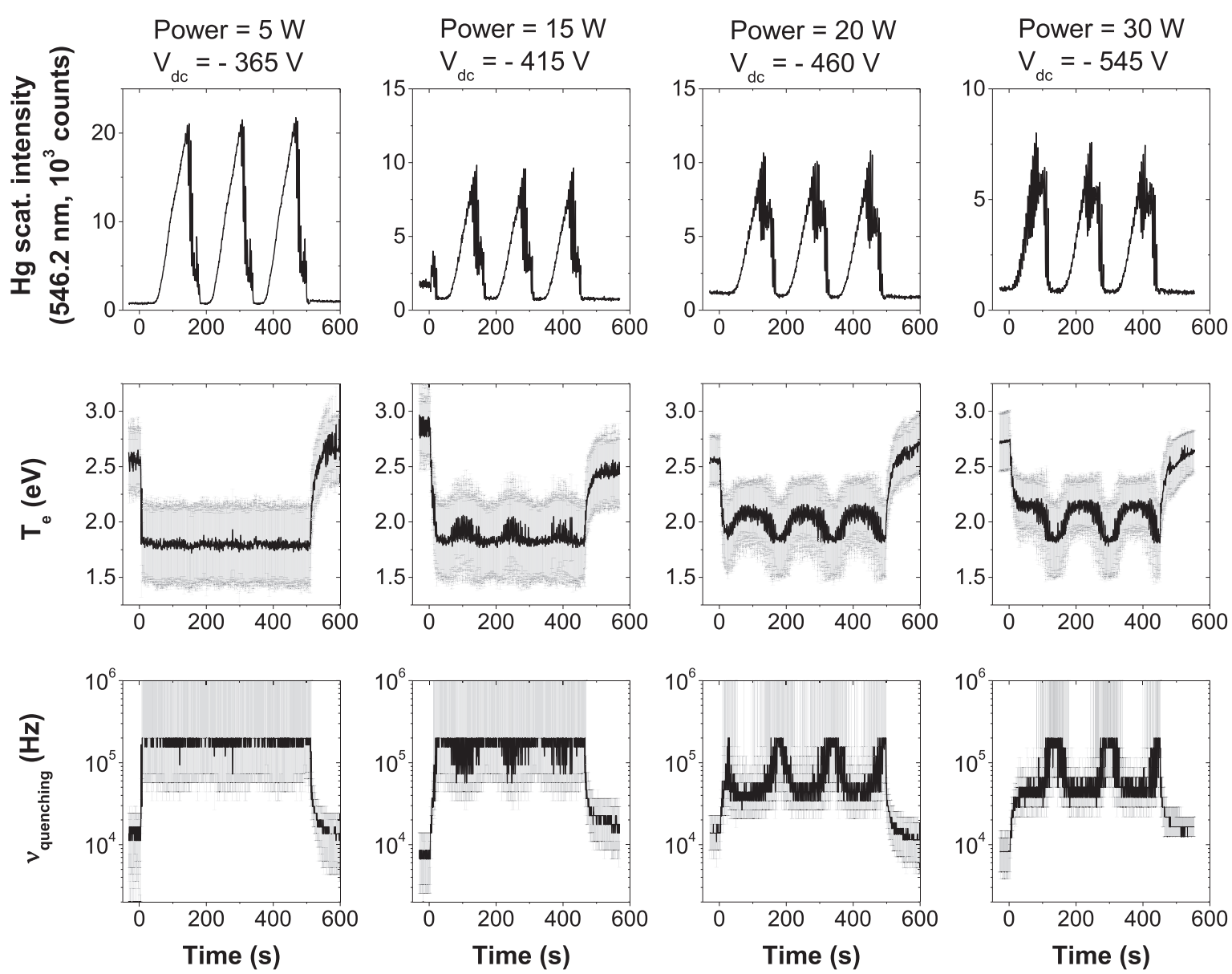

Figure 15. The effect of the HMDSO duty cycle on the temporal development of the Hg scattering intensity at $435.8 \mathrm{~nm}$, the electron temperature and the quenching frequency obtained from the collisional-radiative model of OES spectra recorded in Ar/HMDSO plasma. Error bars are represented in grey. Ar partial pressure $\mathrm{p}_{\mathrm{Ar}}=5.33 \mathrm{~Pa}$; HMDSO duty cycle $=70 \%$.

ionization of HMDSO and its compounds becomes an important source of electrons for the plasma maintenance. The electron temperature experiences strong variations over the dust formation/disappearance cycles. It increases while the dust nucleates and is growing in the plasma and decreases during the dust lost. The electron density shows the opposite trend. This is consistent with previously reported results. Although the a priori obtained variations of the electron density at the frequency of pulsed injection of HMDSO are not significant, the performed FFT analysis shows a trend at the second fundamental frequency, meaning that the HMDSO pulsed injection has an immediate effect on the electron density. The HMDSO duty cycle is found to be the relevant parameter, rather than the HMDSO pulse injection time-on, for the cyclic dust formation in Ar/HMDSO plasmas. The dust formation/disappearance frequency increases linearly with the HMDSO duty cycle. When the amount of injected HMDSO and the fragments it generates in the argon plasma is very high, the upper limit of the quenching frequency is attained and the process of quenching of argon metastable atoms remains saturated. At this stage the periodicity of the main plasma parameters, electron temperature and electron density in time is lost and the plasma maintenance changes. The multi-scale approach applied in this study reveals the straightforward relation between the two fundamental frequencies: the first frequency of the HMDSO pulsed injection, in particular the HMDSO duty cycle, and the second frequency of the dust formation/disappearance cycle in $\mathrm{Ar} /$ HMDSO plasmas.

\section{Acknowledgments}

This work was supported by the Natural Sciences and Engineering Research Council of Canada through both the Discovery Grants Program and the Postgraduate ScholarshipsDoctoral Program. The authors acknowledge support from the European Research Council under the European Union's Seventh Framework Programme ERC-2013-SyG, Grant Agreement no. 610256 NANOCOSMOS. This work has also been supported by the CNRS-UdeM network GDRI 'Nanomatériaux Multifonctionnels Contrôlés-NMC'.

\section{ORCID iDs}

K Makasheva (10 https://orcid.org/0000-0001-6113-3593

L Stafford (i) https://orcid.org/0000-0003-0647-543X 


\section{References}

[1] Mikikian M, Boufendi L, Bouchoule A, Thomas H M, Morfill G E, Nefedov A P and Fortov V E 2003 Formation and behaviour of dust particle clouds in a radio-frequency discharge: results in the laboratory and under microgravity conditions New J. Phys. 19 1-12

[2] Fanelli F, Mastrangelo A M and Fracassi F 2014 Aerosolassisted atmospheric cold plasma deposition and characterization of superhydrophobic organic-inorganic nanocomposite thin films Langmuir 30 857-65

[3] Profili J, Levasseur O, Blaisot J B, Koronai A, Stafford L and Gherardi N 2016 Nebulization of nanocolloidal suspensions for the growth of nanocomposite coatings in dielectric barrier discharges Plasma Process. Polym. 13 981-9

[4] Selwyn G S, Singh J and Bennett R S 1989 In situ laser diagnostic studies of plasma-generated particulate contamination J. Vac. Sci. Technol. A 7 2758-65

[5] Kortshagen U 2009 Nonthermal plasma synthesis of semiconductor nanocrystals J. Phys. D: Appl. Phys. 42113001

[6] Mikikian M and Boufendi L 2004 Experimental investigations of void dynamics in a dusty discharge Phys. Plasmas 11 3733-7

[7] Samsonov D and Goree J 1999 Instabilities in a dusty plasma with ion drag and ionization Phys. Rev. E 59 1047-58

[8] Chai K B, Seon C R, Chung C W, Yoon N S and Choe W 2011 Correlation between nanoparticle and plasma parameters with particle growth in dusty plasmas J. Appl. Phys. 109 013312

[9] Hollenstein C, Dorier J-L, Dutta J, Sansonnens L and Howling A A 1994 Diagnostics of particle genesis and growth in RF silane plasmas by ion mass spectrometry and light scattering Plasma Sources Sci. Technol. 3 278-85

[10] Shiratani M, Kawasaki H, Fukuzawa T, Yoshioka T, Ueda Y, Singh S and Watanabe Y 1996 Simultaneous in situ measurements of properties of particulates in rf silane plasmas using a polarization-sensitive laser-light-scattering method J. Appl. Phys. 79 104-9

[11] Hollenstein C 2000 The physics and chemistry of dusty plasmas Plasma Phys. Control. Fusion 42 R93-104

[12] Mendis D A 2002 Progress in the study of dusty plasmas Plasma Sources Sci. Technol. 11 A219-28

[13] Watanabe Y 2006 Formation and behaviour of nano/microparticles in low pressure plasmas J. Phys. D: Appl. Phys. 39 R329-61

[14] Calafat M, Escaich D, Clergereaux R, Raynaud P and Segui Y 2007 Particle formation in acetylene very low-pressure high density magnetized plasmas Appl. Phys. Lett. 91181502

[15] Cavarroc M, Mikikian M, Tessier Y and Boufendi L 2008 Instabilities during the growth of dust successive generations in silane-based plasmas Phys. Plasmas 15103704

[16] Berndt J, Kovačević E, Stefanović I, Stepanović O, Hong S H, Boufendi L and Winter J 2009 Some aspects of reactive complex plasmas Contrib. to Plasma Phys. 49 107-33

[17] Bouchoule A and Boufendi L 1993 Particulate formation and dusty plasma behaviour in argon-silane RF discharge Plasma Sources Sci. Technol. 2 204-13

[18] Boufendi L and Bouchoule A 1994 Particle nucleation and growth in a low-pressure argon-silane discharge Plasma Sources Sci. Technol. 3 262-7

[19] Winter J, Berndt J, Hong S-H, Kovačević E, Stefanović I and Stepanović O 2009 Dust formation in $\mathrm{Ar} / \mathrm{CH}_{4}$ and $\mathrm{Ar} / \mathrm{C}_{2} \mathrm{H}_{2}$ plasmas Plasma Sources Sci. Technol. 18034010

[20] Massereau-Guilbaud V, Pereira J, Géraud-Grenier I and Plain A 2009 Influence of the power on the particles generated in a low pressure radio frequency nitrogen-rich methane discharge J. Appl. Phys. 105033302

[21] Ricci M, Dorier J, Hollenstein C and Fayet P 2011 Influence of argon and nitrogen admixture in $\mathrm{HMDSO} / \mathrm{O} 2$ plasmas onto powder formation Plasma Process. Polym. 8 108-17

[22] Despax B, Makasheva K and Caquineau H 2012 Cyclic powder formation during pulsed injection of hexamethyldisiloxane in an axially asymmetric radiofrequency argon discharge J. Appl. Phys. 112093302

[23] Despax B, Gaboriau F, Caquineau H and Makasheva K 2016 Influence of the hexamethyldisiloxane temporal variations on the cyclic dust formation in hexamethyldisiloxane-argon radiofrequency discharge: analysis by progressive in time mass spectrometry AIP Adv. 6105111

[24] Roth C, Oberbossel G, Buitrago E, Heuberger R and Rudolf Von Rohr P 2012 Nanoparticle synthesis and growth in a continuous plasma reactor from organosilicon precursors Plasma Process. Polym. 9 119-34

[25] Pattyn C, Kovacevic E, Hussain S, Dias A, Lecas T and Berndt J 2018 Nanoparticle formation in a low pressure argon/aniline RF plasma Appl. Phys. Lett. 013102

[26] Inagaki N 1996 Plasma Surface Modification and Plasma Polymerization (Boca Raton: CRC Press)

[27] Maex K, Baklanov M R, Shamiryan D, Iacopi F, Brongersma S H and Yanovitskaya Z S 2003 Low dielectric constant materials for microelectronics J. Appl. Phys. 93 8793-841

[28] Hilt F, Gherardi N, Duday D, Berné A and Choquet P 2016 Efficient flame retardant thin films synthesized by atmospheric pressure PECVD through the high Codeposition rate of hexamethyldisiloxane and triethylphosphate on polycarbonate and polyamide-6 substrates ACS Appl. Mater. Interfaces 8 12422-33

[29] Wang H, Lin J and Shen Z X 2016 Polyaniline (PANi) based electrode materials for energy storage and conversion $J$. Sci. Adv. Mater. Devices 1 225-55

[30] Levasseur O, Stafford L, Gherardi N, Naudé N, Blanchard V, Blanchet P, Riedl B and Sarkissian A 2012 Deposition of hydrophobic functional groups on wood surfaces using atmospheric-pressure dielectric barrier discharge in heliumhexamethyldisiloxane gas mixtures Plasma Process. Polym. 9 1168-75

[31] Praburam G and Goree J 1996 Experimental observation of very low-frequency macroscopic modes in a dusty plasma Phys. Plasmas 31212

[32] Mikikian M, Couëdel L, Cavarroc M, Tessier Y and Boufendi L 2007 Self-excited void instability in dusty plasmas: plasma and dust cloud dynamics during the heartbeat instability New J. Phys. 9 268-268

[33] Johnson E V, Djeridane Y, Abramov A and Roca i Cabarrocas P 2008 Experiment and modelling of very low frequency oscillations in RF-PECVD: a signature for nanocrystal dynamics Plasma Sources Sci. Technol. 17035029

[34] Boufendi L, Gaudin J, Huet S, Viera G and Dudemaine M 2001 Detection of particles of less than $5 \mathrm{~nm}$ in diameter formed in an argon-silane capacitively coupled radiofrequency discharge Appl. Phys. Lett. 79 4301-3

[35] Cavarroc M, Jouanny M C, Radouane K, Mikikian M and Boufendi L 2006 Self-excited instability occurring during the nanoparticle formation in an $\mathrm{Ar}-\mathrm{SiH}_{4}$ low pressure radio frequency plasma J. Appl. Phys. 99064301

[36] Bilik N, Anthony R, Merritt B A, Aydil E S and Kortshagen U R 2015 Langmuir probe measurements of electron energy probability functions in dusty plasmas J. Phys. D: Appl. Phys. 48105204

[37] Kovačević E, Stefanović I, Berndt J and Winter J 2003 Infrared fingerprints and periodic formation of nanoparticles in $\mathrm{Ar} / \mathrm{C}_{2} \mathrm{H}_{2}$ plasmas J. Appl. Phys. 93 2924-30

[38] Calafat M, Yuryev P, Drenik A, Slim A and Clergereaux R 2011 Carbon nanoparticle/hydrogenated amorphous carbon composite thin films formed in ECR plasma Plasma Process. Polym. 8 401-8 
[39] Deschenaux C, Affolter A, Magni D, Hollenstein C and Fayet $\mathrm{P} 1999$ Investigations of $\mathrm{CH}_{4}, \mathrm{C}_{2} \mathrm{H}_{2}$ and $\mathrm{C}_{2} \mathrm{H}_{4}$ dusty RF plasmas by means of FTIR absorption spectroscopy and mass spectrometry J. Phys. D: Appl. Phys. 32 1876-86

[40] Dap S, Lacroix D, Hugon R and Bougdira J 2013 Retrieving particle size and density from extinction measurement in dusty plasma, Monte Carlo inversion and ray-tracing comparison J. Quant. Spectrosc. Radiat. Transf. 128 18-26

[41] Dap S, Lacroix D, Patisson F, Hugon R, De Poucques L and Bougdira J 2010 Agglomeration processes in carbonaceous dusty plasmas, experiments and numerical simulations New J. Phys. 12093014

[42] Barbosa S et al 2016 An introduction to light extinction spectrometry as a diagnostic for dust particle characterisation in dusty plasmas J. Plasma Phys. 82615820403

[43] Barbosa S, Couëdel L, Arnas C, Kumar K K, Pardanaud C and Onofri F R A 2016 In-situ characterisation of the dynamics of a growing dust particle cloud in a direct-current argon glow discharge J. Phys. D: Appl. Phys. 49045203

[44] Gordillo-Vázquez F J, Camero M and Gómez-Aleixandre C 2006 Spectroscopic measurements of the electron temperature in low pressure radiofrequency $\mathrm{Ar} / \mathrm{H}_{2} / \mathrm{C}_{2} \mathrm{H}_{2}$ and $\mathrm{Ar} / \mathrm{H}_{2} / \mathrm{CH}_{4}$ plasmas used for the synthesis of nanocarbon structures Plasma Sources Sci. Technol. 15 42-51

[45] Chingsungnoen A, Wilson J I B, Amornkitbamrung V, Thomas $\mathrm{C}$ and Burinprakhon $\mathrm{T} 2007$ Spatially resolved atomic excitation temperatures in $\mathrm{CH}_{4} / \mathrm{H}_{2}$ and $\mathrm{C}_{3} \mathrm{H}_{8} / \mathrm{H}_{2} \mathrm{RF}$ discharges by optical emission spectroscopy Plasma Sources Sci. Technol. 16 434-40

[46] Massereau-Guilbaud V, Géraud-Grenier I and Plain A 2009 Determination of the electron temperature by optical emission spectroscopy in a $13.56 \mathrm{MHz}$ dusty methane plasma: influence of the power J. Appl. Phys. 106113305

[47] Massereau-Guilbaud V, Géraud-Grenier I, Lagrange J-F, Tawidian H and Mikikian M 2013 Electron temperature evolution in a low-pressure dusty RF nitrogen-rich methane plasma IEEE Trans. Plasma Sci. 41 816-21

[48] Garofano V, Stafford L, Despax B, Clergereaux R and Makasheva K 2015 Cyclic evolution of the electron temperature and density in dusty low-pressure radio frequency plasmas with pulsed injection of hexamethyldisiloxane Appl. Phys. Lett. 107183104

[49] Despax B and Raynaud P 2007 Deposition of 'polysiloxane' thin films containing silver particles by an RF asymmetrical discharge Plasma Process. Polym. 4 127-34

[50] Milliere L, Makasheva K, Laurent C, Despax B, Boudou L and Teyssedre G 2016 Silver nanoparticles as a key feature of a plasma polymer composite layer in mitigation of charge injection into polyethylene under dc stress J. Phys. D: Appl. Phys. 49015304

[51] Donnelly V M 2004 Plasma electron temperatures and electron energy distributions measured by trace rare gases optical emission spectroscopy J. Phys. D: Appl. Phys. 37 R217-36

[52] Boivin S, Glad X, Boeuf J-P and Stafford L 2018 Analysis of the high-energy electron population in surface-wave plasma columns in presence of collisionless resonant absorption Plasma Sources Sci. Technol. 27095011
[53] Denysenko I, Yu M Y, Ostrikov K and Smolyakov A 2004 Spatially averaged model of complex-plasma discharge with self-consistent electron energy distribution Phys. Rev. EStat. Physics, Plasmas, Fluids, Relat. Interdiscip. Top. 70 046403

[54] Mewe R 1967 Relative intensity of helium spectral lines as a function of electron temperature and density $\mathrm{Br}$. J. Appl. Phys. 18 107-18

[55] Malyshev M V and Donnelly V M 1999 Trace rare gases optical emission spectroscopy: nonintrusive method for measuring electron temperatures in low-pressure, lowtemperature plasmas Phys. Rev. E 606016

[56] Gangwar R K, Sharma L, Srivastava R and Stauffer A D 2012 Argon plasma modeling with detailed fine-structure cross sections J. Appl. Phys. 111053307

[57] Alexander M R, Jones F J and Short R D 1997 Mass spectral investigation of the radio-frequency plasma deposition of hexamethyldisiloxane J. Phys. Chem. B 101 3614-9

[58] Velazco J E, Kolts J H and Setser D W 1978 Rate constants and quenching mechanisms for the metastable states of argon, krypton, and xenon J. Chem. Phys. 69 4357-73

[59] Piper L G, Velazco J E and Setser D W 1973 Quenching cross sections for electronic energy transfer reactions between metastable argon atoms and noble gases and small molecules Vacuum $593323-40$

[60] Stefanović I, Sadeghi N and Winter J 2010 The influence of $\mathrm{C}_{2} \mathrm{H}_{2}$ and dust formation on the time dependence of metastable argon density in pulsed plasmas J. Phys. D: Appl. Phys. 43152003

[61] Basner R, Foest R, Schmidt M, Becker K and Deutsch H 1998 Absolute total and partial electron impact ionization cross sections of hexamethyldisiloxane Int. J. Mass Spectrom. 176 $245-52$

[62] Jiao C Q, DeJoseph C A and Garscadden A 2005 Ion chemistries in hexamethyldisiloxane J. Vac. Sci. Technol. A 23 1295-304

[63] von Keudell A, Hopf C, Jacob W and von Keudell A 1999 Surface loss probabilities of hydrocarbon radicals on amorphous hydrogenated carbon film surfaces: consequences for the formation of re-deposited layers in fusion experiments $\mathrm{Nucl}$. Fusion 39 1451-62

[64] Jauberteau J L and Jauberteau I 2012 Comparison of hexamethyldisiloxane dissociation processes in plasma J. Phys. Chem. A 116 8840-50

[65] Tang X-Z and Luca Delzanno G 2014 Orbital-motion-limited theory of dust charging and plasma response Phys. Plasmas 21123708

[66] Gatti M and Kortshagen U 2008 Analytical model of particle charging in plasmas over a wide range of collisionality Phys. Rev. E 78046402

[67] Fukuzawa T, Shiratani M and Watanabe Y 1994 Novel in situ method to detect subnanometer-sized particles in plasmas and its application to particles in helium-diluted silane radio frequency plasmas Appl. Phys. Lett. 64 3098-100

[68] Godyak V A and Piejak R B 1990 Abnormally low electron energy and heating-mode transition in a low-pressure argon rf discharge at $13.56 \mathrm{MHz}$ Phys. Rev. Lett. 65 996-9 